Complementary resistive switching devices are engineered by sandwiching two ferroelectric tunnel barriers in between nanometric metallic electrodes. Stored data can be read either using destructive readout protocols, as in early complementary resistive switching devices, or more interestingly, non-destructively by simply selecting the reading voltage. Data shows that the engineered devices are energetically more efficient that single ferroelectric tunnel barriers.

Keyword: complementary resistive switching

M. Qian, I. Fina,* F. Sánchez, J. Fontcuberta*

Complementary resistive switching using metal-ferroelectric-metal tunnel junctions

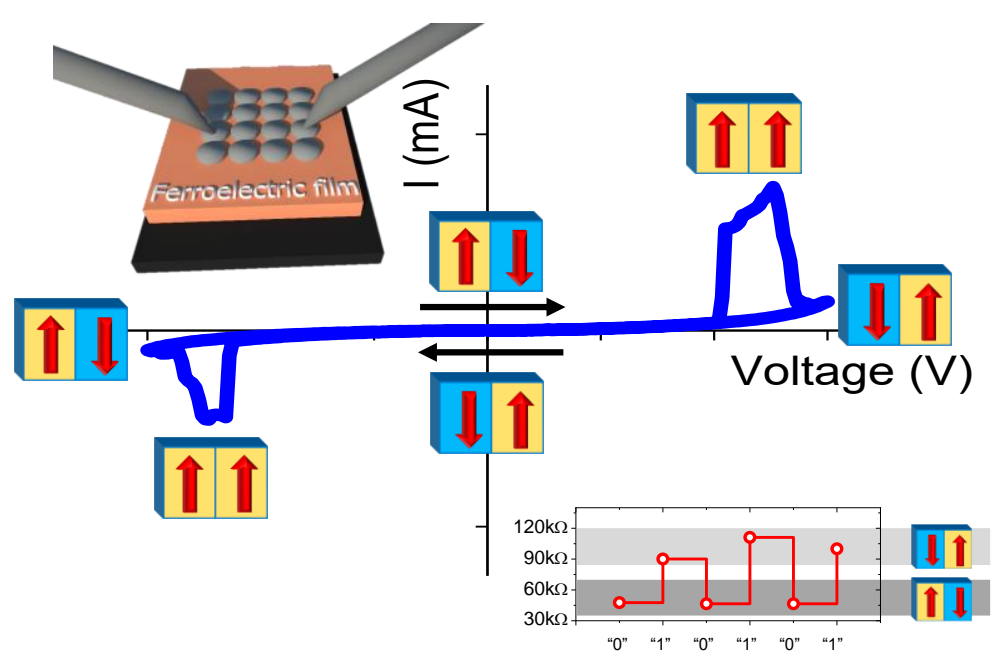




\section{Complementary resistive switching using metal-ferroelectric-metal tunnel junctions}

Mengdi Qian, Ignasi Fina, * Florencio Sánchez, and Josep Fontcuberta*

M. Qian, I. Fina,* F. Sánchez, J. Fontcuberta*

Institut de Ciència de Materials de Barcelona (ICMAB-CSIC), Campus UAB, Bellaterra

08193, Barcelona, Spain

E-mail: ifina@icmab.es; fontcuberta@icmab.cat

Keywords: ferroelectric, complementary resistive switching, ferroelectric tunnel junctions, $\mathrm{BaTiO}_{3}$

Complementary resistive switching devices are receiving attention because they can potentially solve the current-sneak and current-leakage problems of memory arrays based on resistive switching elements. Here we show that the simple anti-serial connection of two ferroelectric tunnel junctions, based on $\mathrm{BaTiO}_{3}$, with symmetric top metallic electrodes and a common, floating bottom nanometric film electrode, constitute a complementary resistive switching (CRS) memory element. It allows non-volatile storage of binary states ("1" = "HRS-LRS" and "0" = "LRS- HRS"), where HRS(LRS) indicate the high(low) resistance state of each ferroelectric tunnel junction. Remarkably, these states have an identical and large resistance in the remanent state, characteristic of CRS. We show writing capability and, depending on the reading voltage amplitude, non-destructive or destructive reading schemes can be chosen. Moreover, we experimentally demonstrate that this dual-tunnel device has a significantly lower power consumption than the single ferroelectric tunnel junction to perform writing/reading functions. These findings illustrate that the recent impressive development of ferroelectric tunnel junctions can be further exploited to contribute solving a critical bottleneck of data storage and logic functions based on resistive switching elements. 


\section{Introduction}

Large changes of electric resistance can be observed in some thin-film devices when stressed by electric signals, for instance electric current or voltage. The resulting resistive switching (RS) is observed in large variety of materials and heterostructures and different mechanisms have been shown to be at play, depending on the precise nature of materials and measuring conditions. The RS devices (sometime also loosely named, memristive devices) are two terminal devices basically consisting on a rather simple trilayer structure composed by a suitable thin film sandwiched in between two metallic electrodes. Two non-volatile resistance states, low-resistance state (LRS) and high resistance state (HRS), can be obtained by a suitable electric stimulus. These two states, can be used to store logic information ("0" and "1"). The fact that the information is stored in the electrical resistance, implies that in principle, RS devices can be scaled down as required by the most aggressive scaling in electronic industry. This is an important advantage compared with electronic memory devices based on electric charge storage. ${ }^{[1-3]}$ In fact, the resistive random access memory (RRAM) elements are intensively investigated on the path towards higher memory density and enhanced computing performance as required by present and future information technologies. The so-called passive crossbar array of RS devices constitutes the backbone of resistive random access memories and reconfigurable logics and holds promises to become central in the quest for fusion of logic and memory functions and neuromorphic computing. ${ }^{[4-7]}$ The crossbar array consists of an array of parallel bottom metallic electrodes and a perpendicular array of top metallic electrodes (so called, word and bit lines) that sandwich at each crossing point, the RS element. By applying suitable voltage at the specified word and bit lines, the state of a RS element is set to LRS (ON) or HRS (OFF). Inherent to this array configuration is that the bottom electrode connects all elements in a row and the top electrode connects all elements in a column. It thus follows that: i) if several elements are in LRS state, 
when current is used to probe the state of a particular element, charge will leak across all LRS elements (sneak current) thus compromising the reading and ii) the presence of LRS states implies a permanent current leakage and a concomitant Joule dissipation. These intrinsic bottlenecks of crossbar arrays are well recognized and several approaches, such as integration of strongly rectifying elements, ${ }^{[8]}$ were proposed to overcome this drawback. In 2010, E. Linn et al. ${ }^{[9]}$ proposed a simple solution to solve the sneak problem: connect two RS devices in anti-serial configuration in such a way that if one is in LRS state the other is in the HRS state (so-called complementary resistive switching, CRS). In these circumstances, information is stored not in the state of a single RS element but in the state of distinguishable coupled pair: LRS-HRS and HRS-LRS, representing different logic states (“0” and " 1 "). Of relevance is that the resulting coupled state is always in a high resistance state at remanence and thus the sneak and leakage currents are reduced.

This novel approach was successfully demonstrated using a variety of materials and resistive switching mechanisms. For instance, in $\mathrm{Pt} / \mathrm{SiO}_{2} / \mathrm{GeSe} / \mathrm{Cu},{ }^{[9]}$ and in all-oxides RRAMs, ${ }^{[10-16]}$ where logic functions were also implemented. In general, in this approach the data reading is achieved by applying a voltage pulse large enough to switch one of the RS elements from HRS to LRS and reading the subsequent current. This readout mechanism is destructive as it switches one of the memory elements and thus a rewriting pulse step is required to restore the information. Therefore, the requirements on device endurance become even more stringent. Ferroelectric tunnel junctions are being considered for its implementation as RS elements. In ferroelectric tunnel junctions, electric-field biasing allows modifying the polarization state of the ferroelectric, giving rise to distinguishable resistance states of the tunnel barrier, ${ }^{[17-32]}$ not only useful for memory applications but also for logic operations. ${ }^{[33]}$ It was reported that not only purely electronic mechanism (polarization reversal) but also ionic motions can contribute to the resistive switching states of ferroelectric tunnel junctions $\left(.^{[17,23,34-38]}\right.$ Anyhow, if 
ferroelectric memory elements are used in crossbar geometry, the current sneak problem remains as a serious bottleneck.

To overcome this challenge, $\mathrm{Xi}$ et al. ${ }^{[39]}$ took advantage of the strongly rectifying nature of the Schottky barrier between a ferroelectric $\left(\mathrm{BaTiO}_{3}, \mathrm{BTO}\right)$ and single crystal of a semiconducting electrode ( $\mathrm{Nb}$ doped $\left.\mathrm{SrTiO}_{3}, \mathrm{Nb}: \mathrm{STO}\right)$, to create a bulky CRS device. Using this approach, the authors successfully demonstrated a significant suppression of the sneakcurrent and a non-destructive readout. However, from a practical point of view, the use of a semiconducting $\mathrm{Nb}$ :STO single crystals as electrode hampers the integration of this structure in technological processes. On the other hand, the growth of Nb:STO films with well controlled dopant concentration and optimal properties is extremely challenging, ${ }^{[40,41]}$ and requires harsh conditions. ${ }^{[42]}$ In addition, atomically flat and highly conducting Nb:STO films, as required for using as bottom electrode in tunnel devices, were only reported using STO substrates. ${ }^{[41-43]}$ Therefore, the use of Nb:STO seems to limit potential applications. In addition, CRS devices based on ferroelectric tunnel junctions with Schottky-barrier-controlled responses show gradual HRS/LRS and LRS/HRS transitions rather than abrupt as it would be required for optimal suppression of the sneak current issue. ${ }^{[39]}$ Therefore, other approaches are required to create integrable ferroelectric-based CRS.

Here we show that by using a simple arrangement of two nanometric metallic electrodes, $\mathrm{Pt}$ and $\mathrm{La}_{2 / 3} \mathrm{Sr}_{1 / 3} \mathrm{MnO}_{3}$ (LSMO) sandwiching a ferroelectric BTO epitaxial layer to form a tunnel junction, a thin CRS device can be obtained, allowing either non-destructive or destructive readout protocols. As LSMO, as well as other metallic oxides, can be used as nanometric templates to grow epitaxial BTO on several single crystalline substrates, including Silicon, ${ }^{[44-}$ ${ }^{46]}$ the results reported here approach the ferroelectric CRS device concept to practical realization. The current-voltage characteristics of the CRS device reported here shows sharp switching between LRS/HRS and HRS/LRS states similar to $\mathrm{Pt} / \mathrm{SiO}_{2} / \mathrm{GeSe}_{\mathrm{Cu}}{ }^{[9]}$ and a 
substantially improved power budget compared with single ferroelectric tunnel junction devices.

\section{Results and Discussion}

Ferroelectric BTO $(\approx 4 \mathrm{~nm})$ tunnel devices using LSMO as bottom electrode and Pt as top electrode are fabricated on $\left(\mathrm{LaAlO}_{3}\right)_{0.3}\left(\mathrm{Sr}_{2} \mathrm{TaAlO}_{6}\right)_{0.7}(001)$ (LSAT) substrate. Several top Pt contacts are prepared in the same sample, thus several tunneling devices are available for testing, as shown in Figure 1(a). Current-voltage (I-V) electric measurements are performed using two different configurations. In the first configuration, a ferroelectric junction is tested by applying a bias voltage $(V)$ on a Pt electrode, contacted with a micrometric needle [Figure 1(a)], while the bottom electrode (LSMO) is grounded using another needle contacted at the edge of the sample, in the so-called "top-bottom" (T-B) configuration (sketch in Figure 1(b)). In this case, for a positive $V$ bias, the electric current flows from a top Pt electrode to the bottom LSMO electrode [as indicated by the blue line in Figure 1(b)], thus measuring the Pt/BTO/LSMO structure [enclosed by dashed line ini Figure 1(b)]. Oppositte for negative $V$ bias. In the second configuration, two ferroelectric junctions are contacted through two top Pt electrodes [Figure 1(c)], one of them is biased with $V$ and the other is grounded. This is the so-called "top-top" (T-T) configuration and it is sketched in Figure 1(d). In this case, the electric current flows from one top Pt electrode to another top Pt electrode via the bottom LSMO electrode, crossing twice the BTO layer [as indicated by the blue line in Figure 1(d)]; this allow measuring a Pt/BTO/LSMO/BTO/Pt structure, which corresponds to the anti-serial connection of two Pt/BTO/LSMO capacitors [both enclosed by dashed lines in Figure 1(d)].

In Figure 2(a), we show an $I-V$ curve recorded in T-B configuration. It can be appreciated that the $I-V$ curve is clockwise in the $V>0$ range and anticlockwise in the $V<0$ range. In the $V>0$ region, a switch from the low-resistance state (LRS) to the high resistance state (HRS) occurs at a threshold voltage, known as reset voltage ( $\left.\mathrm{V}_{\text {RESET }}\right)$. This switching process is associated to the reversal of the ferroelectric polarization $\mathrm{P}$ of the barrier from $\mathrm{P}^{\uparrow}$ to $\mathrm{P}^{\downarrow}$, where 
$\uparrow$ and $\downarrow$ indicate the pointing direction of the polarization (towards Pt and LSMO respectively), ${ }^{[21,23]}$ and the concomitant modification of the height and width of the tunnel barrier. In Supp. Inform. Figure S1, we include the analysis of the barrier properties using the Brinkman ${ }^{[47]}$ model. It is worth noticing that the barrier parameters extracted from the I-V curves in this $\mathrm{Pt} / \mathrm{BTO} / \mathrm{LSMO} / / \mathrm{LSAT}$ junctions are very similar to those reported for similar $\mathrm{Pt} / \mathrm{BTO} / \mathrm{LSMO} / / \mathrm{STO}$ devices, ${ }^{[21,31]}$ indicating that using different substrates may affect the coercive field but it does not affect the barrier characteristics. Indeed, the coercive voltage of BTO/LSMO//LSAT, and thus $V_{S E T}$ and $V_{R E S E T}$, is found here to be larger than in BTO/LSMO//STO.$^{[21,23,48]}$ We ascribe the large coercive voltage to the fact that the BTO film grown on LSAT suffers from a larger in-plane strain $\left(\mathrm{a}_{\text {in-plane, } \mathrm{LSAT}}=3.868 \AA\right.$ $)$ compared to that occurring in films grown on STO (ain-plane, LSAT $=3.905 \AA$ ). This effect should result in an enhanced BTO tetragonality for films grown on LSAT, ${ }^{[49]}$ which would result in larger coercive field. ${ }^{[50]}$ In the $V<0$ region, a switch from the HRS to the LRS occurs at $V_{S E T}$, where $\mathrm{P}^{\downarrow}$ switches to $\mathrm{P}^{\uparrow}$. Although here pure electronic effects, related to polarization reversal, are argued to be at the origin of the observed resistive switching, other mechanisms such as ion migration can also contribute. ${ }^{[51]}$ The results obtained in the T-T configuration, to be described in the following, are performed using the anti-serial connection of A and B junctions, implying that the $V$-biasing of each junction is of opposite polarity with respect to the Pt-LSMO electrodes. In Figure 2(b) we show the $I-V$ characteristics of a reversely biased B junction. Notice that because this B junction is $V$-biased with opposite polarization, the $V>$ 0 region is anticlockwise and the $V<0$ region is clockwise. Consequently, in B junction, the HRS to LRS switch occurs at $V_{S E T}>0$ and the LRS to HRS occurs at $V_{R E S E T}<0$.

In Figure 2(c), we show an illustrative $I-V$ curve of the $\mathrm{AB}$ device measured using two top $\mathrm{Pt}$ electrodes (T-T configuration). Data is recorded biasing the top electrode of junction A and grounding the top electrode of junction B. Comparing data in Figure 2(c) with data in Figures 1(a) and 1(b), one can immediately appreciate the dramatically dissimilar shapes of the 
recorded $I-V$ curves of $\mathrm{A}$ or $\mathrm{B}$ and $\mathrm{AB}$ devices. Two fundamental differences emerge: (i) at remanence, that is at $V=0$, the $\mathrm{AB}$ device is always in a high resistance state (HRS $\left.\mathrm{HRS}_{\mathrm{C}}\right)$, and (ii) two well defined voltage $V_{t h l}$ and $V_{t h 2}$ are observed at $V>0$, where the AB device switches to a low resistance state $\left(\mathrm{LRS}_{\mathrm{CRS}}\right)$ and recovers the $\mathrm{HRS}_{\mathrm{CRS}}$ stat, respectively. Similarly, at $V$ $<0$, a transition from the HRS $\mathrm{CRS}_{\mathrm{C}}$ to $\mathrm{LRS}_{\mathrm{CRS}}$ states occurs at $V_{t h 3}$ while the $\mathrm{HRS}_{\mathrm{CRS}}$ is recovered at $V_{t h 4}$. Overall, the shape of the $I-V$ curve shown in Figure 2(c) is identical to that observed the CRS devices exploiting either valence change or electrochemical metallizationinduced RS responses. ${ }^{[9,16,52,53]}$ In Supp. Inform. Figure S2, the $I-V$ characteristics of the AB device is shown in logarithmic scale. We stress that $I-V$ curves similar to those shown in Figures 1 are also obtained in other junctions and junctions pairs on the same sample (Supp. Inform. Figure S3). In Supp. Inform. Figure S4, we show the $I$ - $V$ characteristics for a whole set $\left(\mathrm{HRS}_{\mathrm{A}}-\mathrm{HRS} \mathrm{S}_{\mathrm{B}}, \mathrm{LRS}_{\mathrm{A}}-\mathrm{HRS}_{\mathrm{B}}, \mathrm{HRS}_{\mathrm{A}}-\mathrm{LRS}_{\mathrm{B}}\right.$, and $\left.\mathrm{LRS}_{\mathrm{A}}-\mathrm{LRS}_{\mathrm{B}}\right)$ of initial states, which helps to visualize the impact of the initial states on the shape of the $I$ - $V$ curves.

To understand the features of the $I-V$ curves in Figures 2(c), we argue as follows. Assume that, in accordance with the $I$ - $V$ curves of A and B shown in Figures 2(a) and 2(b), after the $V=-16$ $\mathrm{V}$ poling, the junction A at $V=0$ is in a LRS and consequently B is in HRS (see Table 1). When applying a positive $V(>0)$, in agreement with the $I-V$ curves of Figure 2(a,b), voltage drops basically at B which is the HRS; upon increasing $V$, at some voltage $\left(V_{t h 1}\right)$, the B junction will switch to the LRS whereas A remains at LRS (as far as, no voltage drops at A), thus the current flowing in the system will abruptly increase (this state is marked in red in the table). Note that the HRS to LRS switch occurs only if $\left|V_{S E T}\right|$ is smaller than $\left|V_{R E S E T}\right|$. When reaching the $V_{t h 2}$ threshold, A will switch to the HRS and the current will drop as seen in Figure 2(c). This state - A in HRS and B in LRS- (labelled, $\mathrm{HRS}_{\mathrm{A}}-\mathrm{LRS}_{\mathrm{B}}$ ) is denoted "1" in Table 1. When reducing $V$ back to $V=0$, the $\mathrm{HRS}_{\mathrm{A}}-\mathrm{LRS}_{\mathrm{B}}$ state is preserved. In fact, even reversing the $V$ polarity, if $V>V_{t h 3}$, the state of the device will be preserved. At $V=V_{t h 3}$, according to Figure 2(a), A will switch to LRS and the current across the device will increase 
abruptly as A and B will be both at LRS (this state is marked in red in the table). At $V=V_{t h 4}$, B will switch to the HRS and thus the current will be reduced again. This state -A in LRS and B in HRS-, labelled: $\mathrm{LRS}_{\mathrm{A}}-\mathrm{HRS} \mathrm{S}_{\mathrm{B}}$, is denoted "0" in Table 1. When reducing V back again to $V=0$, the $\mathrm{LRS}_{\mathrm{A}}-\mathrm{HRS}_{\mathrm{B}}$ state is preserved. The key point of our work is that at remanence $(V=$ 0), two different states $\left(\mathrm{LRS}_{\mathrm{A}}-\mathrm{HRS}_{\mathrm{B}}\right.$ and $\left.\mathrm{HRS}_{\mathrm{A}}-\mathrm{LRS}_{\mathrm{B}}\right)$ can be written having a similarly high resistance $\left(\mathrm{HRS}_{\mathrm{CRS}}\right)$ but being physically different $\left(\mathrm{LRS}_{\mathrm{A}}-\mathrm{HRS}_{\mathrm{B}} \neq \mathrm{HRS}_{\mathrm{A}}-\mathrm{LRS}_{\mathrm{B}}\right)$, as emphasized in Table 1.

Having stablished that different states can be written, we turn now to the writing/reading protocols in the CRS, i.e. T-T configuration. In the following the width of the writing and reading pulses is $\tau_{W}=\tau_{R}=1 \mathrm{~ms}$ and the time delay between writing and reading pulses is $\tau_{D}=$ 1 s. In Figure 3(a,b), we show the voltage-pulse train used to write the $\mathrm{LRS}_{\mathrm{A}}-\mathrm{HRS}_{\mathrm{B}}$ and $\mathrm{HRS}_{\mathrm{A}}-\mathrm{LRS} \mathrm{S}_{\mathrm{B}}$ states (black) and the corresponding reading voltage pulses (red, green and blue). For concreteness, we first consider the case in which a $\mathrm{HRS}_{\mathrm{A}}-\mathrm{LRS}_{\mathrm{B}}$ state is settled using the voltage-pulse train shown in Figure 3(a). In Figure 3(c), we show the corresponding measured current pulses. According to Figure 2(c) and Table 1, to write $\mathrm{HRS}_{\mathrm{A}}-\mathrm{LRS}_{\mathrm{B}}$ (which corresponds to "0") a $V>V_{\text {th } 2}$ is required. We used $V_{W}=+16 \mathrm{~V}$ [Figure 3(a)]. In Figure 3(c), one can appreciate that a large current spike (> $5 \mathrm{~mA}$ ) occurs reflecting that during the $0 \mathrm{~V}$ to $16 \mathrm{~V}$ voltage-writing excursion, a transitory $\mathrm{LRS}_{\mathrm{A}}-\mathrm{LRS}_{\mathrm{B}}$ state was settled before reaching $\mathrm{HRS}_{\mathrm{A}}-\mathrm{LRS}_{\mathrm{B}}$ (black voltage pulse). To read the state, a $V_{R}=12 \mathrm{~V}\left(V_{t h 1}<V_{R}<V_{t h 2}\right)$ pulse (red) is applied. As shown in Figure 3(c), a small current pulse $(\approx 100 \mu \mathrm{A})$ (red) is measured, which is fingerprint of the $\mathrm{HRS}_{\mathrm{CRS}}$. To discriminate between $\mathrm{LRS}_{\mathrm{A}}-\mathrm{HRS}_{\mathrm{B}}$ and $\mathrm{HRS}_{\mathrm{A}}-\mathrm{LRS}_{\mathrm{B}}$ we simply compare the output signals obtained using $V_{R}>$ (red pulse) and $V_{R}<0$ (green pulse) [Figure 3(c)]. It is obvious that the measured current is much larger $(\approx 2 \mathrm{~mA})$ when using $V_{R}<$ 0 than for $V_{R}>0$ thus indicating that memory state was changed to a $\mathrm{LRS}_{\mathrm{CRS}}$ by $V_{R}=-12 \mathrm{~V}$. Because of when using $V_{R}<V_{t h 3}$ a $\mathrm{LRS}_{\mathrm{A}}-\mathrm{LRS}_{\mathrm{B}}$ is stablished, this implies that the initial state was $\mathrm{HRS}_{\mathrm{A}}-\mathrm{LRS}_{\mathrm{B}}$. Therefore, the reading is completed. Application of a subsequent $V_{R}$ pulse 
[blue in Figure 3(c)] of the same polarity does not produce any current spike because the $\mathrm{LRS}_{\mathrm{A}}-\mathrm{LRS}_{\mathrm{B}}$ state was already settled. Application of subsequent $V_{W}$ pulses of the same polarity do not change the written $\mathrm{HRS}_{\mathrm{A}}-\mathrm{LRS}_{\mathrm{B}}$ state and thus no spike current occurs, which is contrary to the result observed if $V_{W}$ pulses of opposite polarity are applied (Supp. Inform. Figure S5).

Writing and reading the $\mathrm{LRS}_{\mathrm{A}}-\mathrm{HRS}$ B state (which corresponds to "1") is done similarly, but using opposite polarity voltage pulses. Accordingly, we used $V_{W}=-16 \mathrm{~V}$ [Figure 3(b) (black pulse)] to set $\mathrm{LRS}_{\mathrm{A}}-\mathrm{HRS}_{\mathrm{B}}$. In Figure 3(d), where the measured current is plotted, one can appreciate similar features to those shown in Figure 3(c), but with opposite polarity. These experiments confirm that indeed, $\mathrm{HRS}_{\mathrm{A}}-\mathrm{LRS}_{\mathrm{B}}$ and $\mathrm{LRS}_{\mathrm{A}}-\mathrm{HRS}_{\mathrm{B}}$ states can be written and read, The complex writing/reading protocol described above can be simplified by using always $V_{R}$ of the same polarity and amplitude. We proceed as follows. First, we set a well-defined initial state preparing the device in a the $\mathrm{HRS}_{\mathrm{A}}-\mathrm{LRS}_{\mathrm{B}}$ state by applying a large $\left(\left|V_{W}\right|=16 \mathrm{~V}>\right.$ $\left.\left|V_{t h 2}, V_{t h 4}\right|\right)$ and long $(100 \mathrm{~ms})$ prepoling positive pulse. Positive or negative voltages $\left(V_{W}=+\right.$ 16 and $-16 \mathrm{~V}$ ) are used to set the different $\mathrm{HRS}_{\mathrm{A}}-\mathrm{LRS}_{\mathrm{B}}$ and $\mathrm{LRS}_{\mathrm{A}}-\mathrm{HRS}_{\mathrm{B}}$ states, respectively. Two reading $V_{R}$ pulses of $12 \mathrm{~V}\left(V_{R}>V_{t h l}\right)$ are applied (see the pulse voltage train in Supp. Inform. Figure S6). In Figure 4(a), the current measured during the first and second reading pulses clearly shows the different behavior. If the $\mathrm{HRS}_{\mathrm{A}}-\mathrm{LRS}_{\mathrm{B}}$ state is settled, the (low) current pulses measured during the first and second pulse are similar (blue solid and dashed lines, which fully overlap in the Figure). Instead, if the $\mathrm{LRS}_{\mathrm{A}}-\mathrm{HRS}_{\mathrm{B}}$ is settled, the first reading pulse shows the spike while increasing the $V$ (solid red line), which is absent during the measurement of the second (dashed red line). Therefore, if reading is performed during the first reading pulse, the presence of the spike current can be used to better distinguish between different logic states; unfortunately, this reading scheme has a main drawback: it is a destructive read-out, and producing and intermediate $\mathrm{LRS}_{\mathrm{A}}-\mathrm{LRS}_{\mathrm{B}}$ of high conductivity at 
remanence (Supp. Inform. Figure S7). If reading is performed during the second reading pulse, the reading current is more stable, but the protocol also produces a destructive read-out. Overall, the described writing/reading protocols (where $\left.V_{R}\right\rangle\left|V_{t h 1}, V_{t h 3}\right|$ ), as commonly found in CRS, requires switching of the memory state and thus it is destructive; therefore, a rewriting pulse would be required for practical applications. The $I-V$ characteristics of the $\mathrm{AB}$ device shown in logarithmic scale, displayed in Supp. Inform. Figure S2, reveals that there is a small difference between the current measured while increasing and decreasing $V$ near the 0 V regions. This current difference can be used for non-destructive readout, and that this is the reason why, we turn now to analyze the effect of changing the $V_{R}$ amplitude.

To obtain a non-destructive reading, the reading voltage-pulse amplitude should be smaller than threshold voltages $\left(0<V_{R}<\left|V_{t h 1}, V_{t h 3}\right|\right)$, to avoid HRS $\mathrm{CRS}_{\text {So }}$ to $\mathrm{S}_{\mathrm{CRS}}$ switching of any junction. To illustrate the results of this reading scheme, we show in Figure 4(b) the current measured during the first (solid) and second (dashed) pulses using $V_{R}=2 \mathrm{~V}$, and the same protocol than Figure 4(a), fulfilling the condition $0<V_{R}<\left|V_{t h l}, V_{t h 3}\right|$. It can be also observed that during the first $V_{R}$, the spike current is not visible and the current measured during the first and second pulses fully overlap (thus, first and second current pulses are almost indistinguishable in the Figure). This is consistent with the fact that $V_{R}$ does not set the $\mathrm{LRS}_{\mathrm{A}^{-}}$ LRS $_{B}$ state because its amplitude is below the threshold voltage. Of relevance, is that the current measured (measured with $V_{R}=2 \mathrm{~V}$ ) after $V_{W}=-16 \mathrm{~V}$ [red data in Figures 3(b)], which settles the $\mathrm{LRS}_{\mathrm{A}}-\mathrm{HRS}_{\mathrm{B}}$ state, shows a clearly different value compared with the current (blue) measured after $V_{W}=+16 \mathrm{~V}$, which settles the $\mathrm{HRS}_{\mathrm{A}}-\mathrm{LRS}_{\mathrm{B}}$ state. Thus a subthreshold voltage can be used for reading operation $\left(0<V_{R}<\left|V_{t h 1}, V_{t h 3}\right|\right)$. For $V_{R}<2 \mathrm{~V}$, both states are not distinguishable as shown in Supp. Inform. Figure S8. In Figures 4(c,d) we summarize the resistance states of the different logic states read by destructive $\left(V_{R}=12 \mathrm{~V}\right)$ and nondestructive $\left(V_{R}=2 \mathrm{~V}\right)$ reading voltages. In both cases, the conductance contrast between both 
states is clear. In Supp. Inform. Figure S8, the resistance states read by $V_{R}=1,2,5$ and $12 \mathrm{~V}$ are summarized.

Using the same voltage pulse train as used in Figures 4(a,b), we collect in Figures 5(a,b), the data obtained to study the stability of different states and the duration of the writing pulse required to set a precise state, respectively. With this purpose we explore the role of $\tau_{\mathrm{D}}$ and $\tau_{\mathrm{w}}$ on the corresponding output signals. In Figures 5(a,b), the current measured during the second $V_{R}=+12 \mathrm{~V}$ is plotted. We use the second $V_{R}$ to avoid the presence of current spikes allowing better comparison (see in Supp. Inform. Figure S9 the current measured during the first $V_{R}$ ). In Figure 5(a), we show the measured current after delay times of $\tau_{D}=1 \mathrm{~s}, 1 \mathrm{~min}$ and $1 \mathrm{~h}$ between the $V_{W}$ and $V_{R}$ keeping constant $\tau_{W, R}=1 \mathrm{~ms}$. It can be observed that irrespective of the value of $\tau_{D}$, the two states (blue and red for $\mathrm{HRS}_{\mathrm{A}}-\mathrm{LRS}_{\mathrm{B}}$ and $\mathrm{LRS}_{\mathrm{A}}-\mathrm{HRS}_{\mathrm{B}}$ respectively) are well defined and the measured current does not significantly vary with $\tau_{\mathrm{D}}$. Thus, we conclude that both states are non-volatile for the explored $\tau_{D}$ range. In Figure 5(b), we show the current measured during the reading pulse, after writing pulses (blue for positive and red for negative) of duration $\tau_{W}=0.1,1,5,10$ and $100 \mathrm{~ms}$ keeping constant $\tau_{D}=1 \mathrm{~s}$ and $\tau_{\mathrm{R}}=1$ ms. It can be appreciated that the conductance contrast measured during the second $V_{R}$ between the two different states, starts to increase at $\approx 1 \mathrm{~ms}$ and keep increasing up to about 10 ms, where the resistance contrasts gradually saturates (see in Supp. Inform. Figure S10 the current measured during the first $V_{R}$ ). Therefore, it follows that relatively slow pulses (about 1 ms) are needed to write the information. This time scale is several orders of magnitude longer than the switching time of a ferroelectric film thus suggesting that other effects are determining the observed slow time-response. A hint into its physical origin can be obtained by considering the time constant $\left(\tau=R C\right.$ ) of our measuring circuit. Using $\mathrm{HRS}_{\mathrm{CRS}} \approx 1 \mathrm{M} \Omega$ (measured at $12 \mathrm{~V}$ ) and a measured capacitance of $C \approx 3 \mathrm{pF}(100 \mathrm{kHz})$, it turns out that $\tau \approx 1$ 
ms, which is in good agreement with the experimental observation. Thus, the observed slow response of our device is attributed to the long time constant $(\approx 1 \mathrm{~ms})$ of our circuit.

Finally, we analyze the power dissipated in the T-T configuration, equivalent to a CRS device, to perform a writing/reading process. With this aim, we apply a long write/read pulse of amplitude $V_{W}= \pm 16 \mathrm{~V}$ and $V_{R}=12 \mathrm{~V}$, as indicated in Figure 6 (see in Supp. Inform. Figure S11, the writing and reading pulse trains). In Figure 6(a), we show the write/read power consumption $(P=I \cdot V)$ for the defined pulse train. It is evident that the writing process requires the largest power. The significance of this power consumption can better appreciated when compared to the power dissipated to perform a similar writing/reading process using the T-B configuration. In T-B configuration, which is in fact the measurement of a single ferroelectric junction, we used $V_{W}= \pm 15$ and $V_{R}=0.1$ V. In Figure 6(b), where we show the corresponding power. Data reveal that in the T-B configuration, the power dissipated during the writing is also larger than during reading, as already observed for T-T. By comparing the power dissipated during the $4^{\text {th }}, 5^{\text {th }}$, and $6^{\text {th }}$ negative writing pulses and the $1^{\text {st }}, 2^{\text {nd }}$, and $3^{\text {rd }}$ for positive writing pulses, it can be appreciated that the power dissipated is for positive writing pulses larger than for negative pulses due to the junction asymmetry. Importantly, the comparison of the power used in writing/reading operations in T-T and T-B [Figure 6(a) and Figure 6(b), respectively] shows that the power consumed in T-T configuration is about one order of magnitude smaller than in T-B, in agreement with earlier findings using nonferroelectric CRS elements. ${ }^{[54]}$ This have the exception of settling the HRS if HRS is already written. In T-B this corresponds to the $2^{\text {nd }}$ and $3^{\text {rd }}$ negative writing pulses of Figure $6(\mathrm{~b})$, where the power is near $30 \mathrm{~mW}$. In the equivalent pulses in T-T [Figure 6(a)] the power is near $20 \mathrm{~mW}$. These similar power values arises because in both cases the LRS and the LRS $\mathrm{CRS}$ states, for T-B and T-T, respectively, are not settled or crossed. Note that the absolute values of the power consumption for data writing in our device is relatively large $(\approx 10 \mathrm{~mW})$. However, as this value scales with device size, it is expected to be reduce by about $10^{-6}$ if 
device size is reduced from about the $10 \times 10 \mu \mathrm{m}^{2}$ as used here to $\approx 10 \times 10 \mathrm{~nm}^{2}$ required in practical implementations, thus illustrating the potential competitiveness of ferroelectricbased CRS.

\section{Conclusion}

The results presented here demonstrate that using BTO ferroelectric tunnel barriers and nanometric thin film electrodes, the two different resistive states, HRS and LRS determined and controlled by the polarization reversal, can be used as building blocks for a CRS device. Here, the CRS is fabricated by using two top metallic contacts on a ferroelectric layer grown on a suitable bottom thin film electrode; this is a simpler structure than the complex metal/solid-electrolyte/metal layers required in some earlier designs of CRS. ${ }^{[9]}$ The anti-serial connection of two BTO tunnel barriers allows to obtain I-V characteristics analogous to those reported in other CRS devices. ${ }^{[9,16,52,53]}$ We show that according to the expected properties of a CRS device, two logic sates "1" and "0", corresponding to the HRS-LRS and LRS-HRS states of the ferroelectric tunnel barriers can be written. The information is stored in a high resistance state, thus avoiding the current-sneak problem, and either non-destructive or destructive reading schemes can be used to retrieve information. We note that the relative change of resistance between the HRS and LRS states is somewhat smaller than in earlier reports, either using Nb:STO single crystals ${ }^{[39]}$ or $\mathrm{LSMO}^{[21]}$ as bottom electrodes; this difference is related to the larger size of the electrodes used in this proof-of-concept approach and accordingly, scaling down should allow to increase the resistance contrast. We also show that using the CRS device, the writing/reading power consumption is reduced by about one order of magnitude compared to that that required to perform the same tasks using a single ferroelectric tunnel barrier. Reducing device footprint down to tens of nanometers and reducing the writing time, here limited by the time constant of the measuring circuit rather than by the intrinsic switching speed of the ferroelectric barrier, may lead to a dramatic reduction of power and energy consumption. Moreover, as polarization switching in the 
ferroelectric barrier is a process that does not imply ionic migration across the barrier, it is expected that endurance would be less compromised that in other resistive switching operational principles. Overall, our simple Pt/ferroelectric/metal/ferroelectric/Pt structure appears to be of potential use in resistive crossbar memory arrays. We strength that BTO/LSMO structure can be epitaxially grown on several single crystalline substrates including Silicon, appropriately buffered with metallic thin films , ${ }^{[44-46]}$ suggesting that BTObased CRS devices can be ultimately fully integrated on silicon. The results described here can be combined with the recent development of ferroelectric $\mathrm{HfO}_{2}$ thin films, grown using CMOS compatible technologies, to develop fully integrable ferroelectric-based complementary switching devices.

\section{Experimental section}

Sample growth: Ferroelectric BTO BTO thin films (4 nm thick) are epitaxial grown on (001) LSAT substrates buffered by a thin $\mathrm{La}_{2 / 3} \mathrm{Sr}_{1 / 3} \mathrm{MnO}_{3}$ (LSMO) $(20 \mathrm{~nm}$ ) layer acting as metallic electrode. Films are grown by pulsed laser deposition (PLD) using the conditions discussed elsewhere ${ }^{[55-58]}$. Structural characterization is shown in Supp. Inform. Figure S12. Circular platinum (Pt) electrodes are deposited ex-situ by sputtering using suitable metallic masks to produce top contacts of lateral dimensions $20 \mu \mathrm{m}$ and a thickness of about $20 \mathrm{~nm}$.

Piezoelectric force microscopy experiments performed on a BTO film grown on STO substrates in the same conditions and with the same thickness than that reported here is shown in Supp. Inform. S13. Data are fully compatible with the ferroelectric character of the sample. Electrical characterization: Current-voltage electrical characteristics are recorded by using triangular $V(t)$ pulses generated by a TFAnalyser2000 (Aixacct Co.). The $I-V$ curves are collected using a triangular $V(t)$ signal following the path $-V_{\max } \rightarrow+V_{\max } \rightarrow-V_{\max }$, where $V_{\max }$, is the absolute value of the maximum applied voltage. To write/read the memory state, during the used write/read voltage pulse trains, we use trapezoidal voltage pulses of $3 \cdot \tau$ duration, 
where $\tau$ is the duration (identical) of the rise, plateau, and decay of the trapezoidal signal.

Trapezoidal voltage pulses are used to minimize the displacive current peaks. Experiments are performed using different writing $\left(\tau_{W}=\tau\right)$ and with fix reading times $\left(\tau_{R}=1 \mathrm{~ms}\right)$ as indicated in the text. The time interval between writing and reading is denoted as the delay time $\left(\tau_{D}\right)$.

Electrical contact configuration: Two contact configurations are used. In top-bottom (T-B), the LMSO bottom electrode is grounded and the top $(\mathrm{Pt})$ electrode is biased at appropriate $V$ voltage, as sketched in Figures 1(b). In top-top (T-T) configuration, one top Pt electrode is grounded and another is biased. In the second configuration, two top Pt electrodes are connected, one connected to $V$ and the other grounded, as sketched in Figure 1(d).

\section{Acknowledgements}

Financial support from the Spanish Ministry of Science, Innovation and Universities, through the "Severo Ochoa" Programme for Centres of Excellence in R\&D (SEV-2015-0496) and the MAT2017-85232-R (AEI/FEDER, EU), MAT2014-56063-C2-1-R, and MAT2015-73839JIN projects, and from Generalitat de Catalunya (2017 SGR 1377) is acknowledged. I.F. acknowledges Ramon y Cajal contract RYC-2017-22531. M.Q. is financially supported by China Scholarship Council (CSC) with No. 201406890019. M.Q. work was done as a part of here Ph.D. program in Materials Science at Universitat Autònoma de Barcelona.

\section{References}

[1] R. Waser, Nanoelectronics and Information Technology, 3rd Edition, Wiley-VHC, Weinheim,Germany, 2012.

[2] Emerging Research Devices., International Technology Roadmap For Semiconductors., 2013.

[3] J. Borghetti, G. S. Snider, P. J. Kuekes, J. J. Yang, D. R. Stewart, R. S. Williams, Nature 2010, 464, 873.

[4] Editorial, Nat. Electron. 2018, 1, 261.

[5] D. B. Strukov, G. S. Snider, D. R. Stewart, R. S. Williams, Nature 2008, 453, 80.

[6] M. Prezioso, F. Merrikh-Bayat, B. D. Hoskins, G. C. Adam, K. K. Likharev, D. B. Strukov, Nature 2015, 521, 61.

[7] I. Schuller, R. Stevens, Neuromorphic Computing : From Materials to Systems Architecture, Department Of Energy, Gaithersburg, 2015.

[8] B. S. Kang, S.-E. Ahn, M.-J. Lee, G. Stefanovich, K. H. Kim, W. X. Xianyu, C. B. Lee, Y. Park, I. G. Baek, B. H. Park, Adv. Mater. 2008, 20, 3066.

[9] E. Linn, R. Rosezin, C. Kügeler, R. Waser, Nat. Mater. 2010, 9, 403.

[10] J. Lee, J. Shin, D. Lee, W. Lee, S. Jung, M. Jo, J. Park, K. P. Biju, S. Kim, S. Park, H. Hwang, in Int. Electron Devices Meet., 2010.

[11] W. Banerjee, X. Xu, H. Lv, Q. Liu, S. Long, M. Liu, Adv. Electron. Mater. 2017, 3, 1. 
[12] A. Schönhals, J. Mohr, D. J. Wouters, R. Waser, S. Menzel, IEEE Electron Device Lett. 2017, 38, 449.

[13] Y. Yang, P. Sheridan, W. Lu, Appl. Phys. Lett. 2012, 100, 203112.

[14] Y. C. Bae, A. R. Lee, J. Bin Lee, J. H. Koo, K. C. Kwon, J. G. Park, H. S. Im, J. P. Hong, Adv. Funct. Mater. 2012, 22, 709.

[15] X. Liu, S. M. Sadaf, S. Park, S. Kim, E. Cha, D. Lee, G. Jung, H. Hwang, IEEE Electron Device Lett. 2013, 34, 235.

[16] T. Breuer, A. Siemon, E. Linn, S. Menzel, R. Waser, V. Rana, Adv. Electron. Mater. 2015, $1,1500138$.

[17] Z. Wen, C. Li, D. Wu, A. Li, N. Ming, Nat Mater 2013, 12, 617.

[18] H. Yamada, V. Garcia, S. Fusil, S. Boyn, M. Marinova, A. Gloter, S. Xavier, J. Grollier, E. Jacquet, C. Carrétéro, C. Deranlot, M. Bibes, A. Barthélémy, ACS Nano 2013, 7, 5385.

[19] K. H. Kim, S. Gaba, D. Wheeler, J. M. Cruz-Albrecht, T. Hussain, N. Srinivasa, W. Lu, Nano Lett. 2012, 12, 389.

[20] A. Chanthbouala, A. Crassous, V. Garcia, K. Bouzehouane, S. Fusil, X. Moya, J. Allibe, B. Dlubak, J. Grollier, S. Xavier, C. Deranlot, A. Moshar, R. Proksch, N. D. Mathur, M. Bibes, A. Barthélémy, Nat. Nanotechnol. 2012, 7, 101.

[21] G. Radaelli, D. Gutiérrez, F. Sánchez, R. Bertacco, M. Stengel, J. Fontcuberta, $A d v$. Mater. 2015, 27, 2602.

[22] A. Chanthbouala, V. Garcia, R. O. Cherifi, K. Bouzehouane, S. Fusil, X. Moya, S. Xavier, H. Yamada, C. Deranlot, N. D. Mathur, M. Bibes, A. Barthélémy, J. Grollier, Nat. Mater. 2012, 11, 860.

[23] M. Qian, I. Fina, F. Sánchez, J. Fontcuberta, Adv. Electron. Mater. 2018, 1800407.

[24] Z. Xi, J. Ruan, C. Li, C. Zheng, Z. Wen, J. Dai, A. Li, D. Wu, Nat. Commun. 2017, 8, 15217.

[25] D. Pantel, S. Goetze, D. Hesse, M. Alexe, Nat. Mater. 2012, 11, 289.

[26] D. Pantel, H. Lu, S. Goetze, P. Werner, D. Jik Kim, A. Gruverman, D. Hesse, M. Alexe, Appl. Phys. Lett. 2012, 100, 232902.

[27] X. S. Gao, J. M. Liu, K. Au, J. Y. Dai, Appl. Phys. Lett. 2012, 101, 142905.

[28] Y. W. Yin, M. Raju, W. J. Hu, X. J. Weng, K. Zou, J. Zhu, X. G. Li, Z. D. Zhang, Q. Li, Front. Phys. 2012, 7, 380.

[29] A. Zenkevich, Y. Matveyev, K. Maksimova, R. Gaynutdinov, A. Tolstikhina, V. Fridkin, Phys. Rev. B 2014, 90, 161409.

[30] S. Boyn, S. Girod, V. Garcia, S. Fusil, S. Xavier, C. Deranlot, H. Yamada, C. Carrétéro, E. Jacquet, M. Bibes, A. Barthélémy, J. Grollier, Appl. Phys. Lett. 2014, 104, 2014.

[31] A. Gruverman, D. Wu, H. Lu, Y. Wang, H. W. Jang, C. M. Folkman, M. Y. Zhuravlev, D. Felker, M. Rzchowski, C.-B. B. Eom, E. Y. Tsymbal, Nano Lett. 2009, 9, 3539.

[32] L. Wang, M. R. Cho, Y. J. Shin, J. R. Kim, S. Das, J. G. Yoon, J. S. Chung, T. W. Noh, Nano Lett. 2016, 16, 3911.

[33] T. You, Y. Shuai, W. Luo, N. Du, D. Burger, I. Skorupa, R. H??bner, S. Henker, C. Mayr, R. Sch??ffny, T. Mikolajick, O. G. Schmidt, H. Schmidt, Adv. Funct. Mater. 2014, 24, 3357.

[34] D. J. Kim, H. Lu, S. Ryu, C. W. Bark, C. B. Eom, E. Y. Tsymbal, a. Gruverman, Nano Lett. 2012, 12, 5697.

[35] R. Soni, A. Petraru, P. Meuffels, O. Vavra, M. Ziegler, S. K. Kim, D. S. Jeong, N. a. Pertsev, H. Kohlstedt, Nat. Commun. 2014, 5, 5414.

[36] H. J. Mao, C. Song, L. R. Xiao, S. Gao, B. Cui, J. J. Peng, F. Li, F. Pan, Phys. Chem. Chem. Phys. 2015, 17, 10146.

[37] M. Li, J. Zhou, X. Jing, M. Zeng, S. Wu, J. Gao, Z. Zhang, X. Gao, X. Lu, J.-M. Liu, M. Alexe, Adv. Electron. Mater. 2015, 1, 1500069. 
[38] Y. Heo, D. Kan, Y. Shimakawa, J. Seidel, Phys. Chem. Chem. Phys. 2016, 18, 197.

[39] Z. Xi, C. Zheng, Z. Wen, ACS Appl. Mater. Interfaces 2018, 10, 6024.

[40] K. Fukushima, S. Shibagaki, Thin Solid Films 1998, 315, 238.

[41] S. Kobayashi, Y. Mizumukai, T. Ohnishi, N. Shibata, Y. Ikuhara, T. Yamamoto, ACS Nano 2015, 9, 10769.

[42] K. S. Takahashi, D. Matthey, D. Jaccard, J.-M. Triscone, K. Shibuya, T. Ohnishi, M. Lippmaa, Appl. Phys. Lett. 2004, 84, 1722.

[43] T. Tomio, H. Miki, H. Tabata, T. Kawai, S. Kawai, J. Appl. Phys. 1994, 76, 5886.

[44] J. Lyu, S. Estandía, J. Gazquez, M. F. Chisholm, I. Fina, N. Dix, J. Fontcuberta, F. Sánchez, ACS Appl. Mater. Interfaces 2018, 10, 25529.

[45] J. Lyu, I. Fina, R. Solanas, J. Fontcuberta, F. Sánchez, Sci. Rep. 2018, 8, 495.

[46] M. Scigaj, C. H. Chao, J. Gázquez, I. Fina, R. Moalla, G. Saint-Girons, M. F. Chisholm, G. Herranz, J. Fontcuberta, R. Bachelet, F. Sánchez, Appl. Phys. Lett. 2016, 109, 122903.

[47] W. F. Brinkman, R. C. Dynes, J. M. Rowell, J. Appl. Phys. 1970, 41, 1915.

[48] G. Radaelli, D. Gutiérrez, M. Qian, I. Fina, F. Sánchez, L. Baldrati, J. Heidler, C. Piamonteze, R. Bertacco, J. Fontcuberta, Adv. Electron. Mater. 2016, 2, 1600368.

[49] K. J. Choi, M. Biegalski, Y. L. Li, A. Sharan, J. Schubert, R. Uecker, P. Reiche, Y. B. Chen, X. Q. Pan, V. Gopalan, L.-Q. Chen, D. G. Schlom, C. B. Eom, Science 2004, 306, 1005.

[50] S. P. Beckman, X. Wang, K. M. Rabe, D. Vanderbilt, Phys. Rev. B 2009, 79, 144124.

[51] Q. H. Qin, L. Äkäslompolo, N. Tuomisto, L. Yao, S. Majumdar, J. Vijayakumar, A. Casiraghi, S. Inkinen, B. Chen, A. Zugarramurdi, M. Puska, S. van Dijken, Adv. Mater. 2016, 28, 6852 .

[52] F. Nardi, S. Balatti, S. Larentis, D. Ielmini, in Int. Electron Devices Meet., 2011, p. 31.1.1-31.1.4.

[53] S. Gao, F. Zeng, M. Wang, G. Wang, C. Song, F. Pan, Sci. Rep. 2015, 5, 15467.

[54] S. Schmelzer, E. Linn, U. Bottger, R. Waser, IEEE Electron Device Lett. 2013, 34, 114.

[55] N. Dix, I. Fina, R. Bachelet, L. Fàbrega, C. Kanamadi, J. Fontcuberta, F. Sánchez, Appl. Phys. Lett. 2013, 102, 172907.

[56] F. Liu, I. Fina, R. Bertacco, J. Fontcuberta, Sci. Rep. 2016, 6, 25028.

[57] F. Liu, I. Fina, D. Gutiérrez, G. Radaelli, R. Bertacco, J. Fontcuberta, Adv. Electron. Mater. 2015, 1, 1500171.

[58] F. Liu, I. Fina, G. Sauthier, F. Sánchez, A. M. Rappe, J. Fontcuberta, ACS Appl. Mater. Interfaces 2018, 10, 23968. 

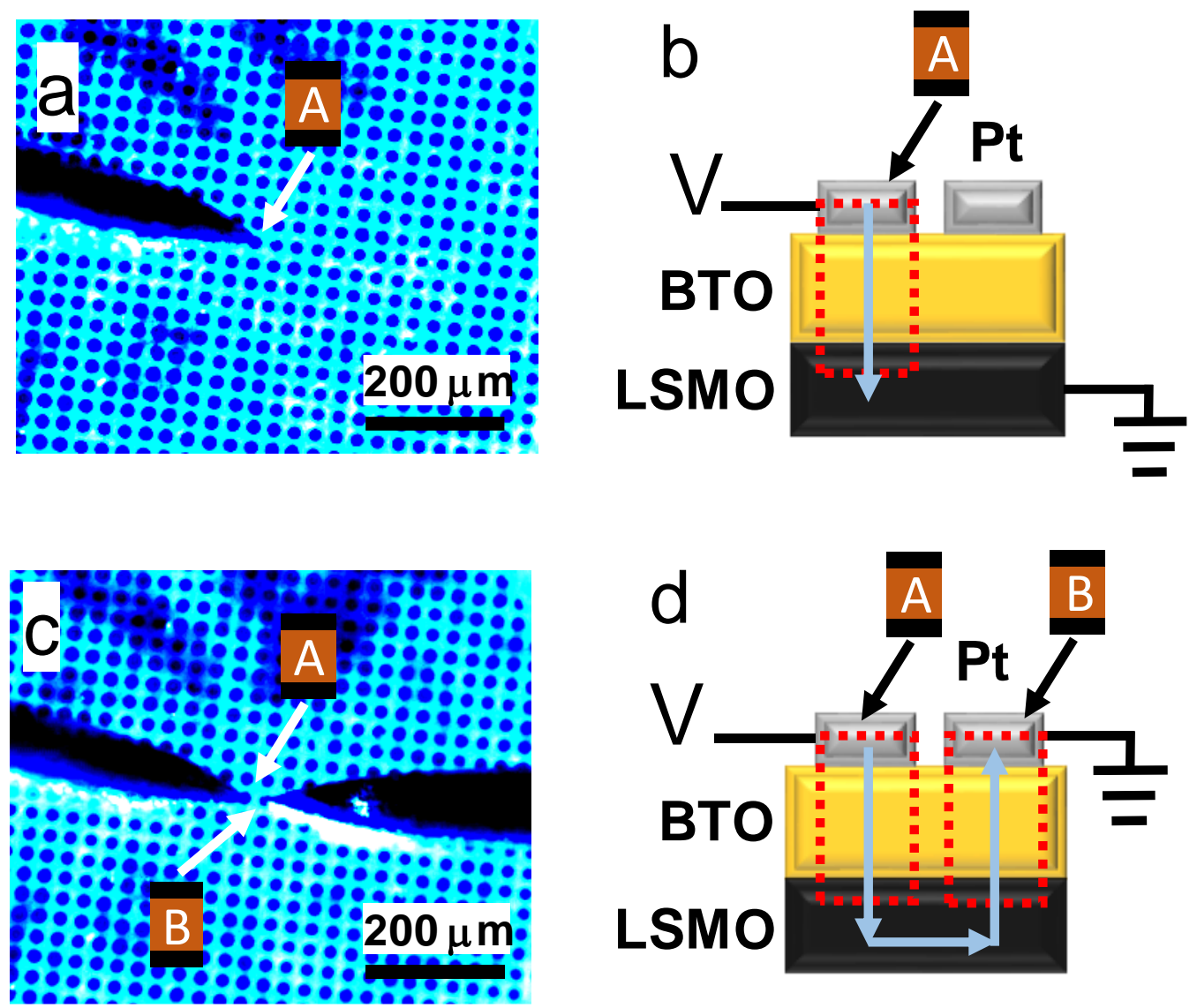

Figure 1. (a) Top view of the sample contacted in T-B configuration. A large number of Pt contacts can be identified as well as the needle used for contacting. (b) Sketch of the electrical connection in the T-B configuration. (c) Top view of the sample contacted in T-T configuration. (d) Sketch of the electrical connection in the T-T configuration. In (b,d), the blue arrows denote the current paths or the electric field direction, while measuring the current and/or applying a voltage $(\mathrm{V}>0)$. Dashed lines denote the measured capacitor structures in each configuration: in (c) the Pt/BTO/LSMO capacitor and in (d) the anti-serial connection of the Pt/BTO/LSMO and LSMO/BTO/Pt. 
a

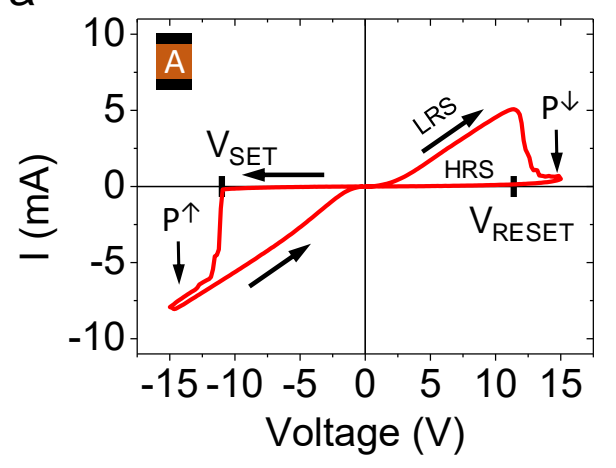

C

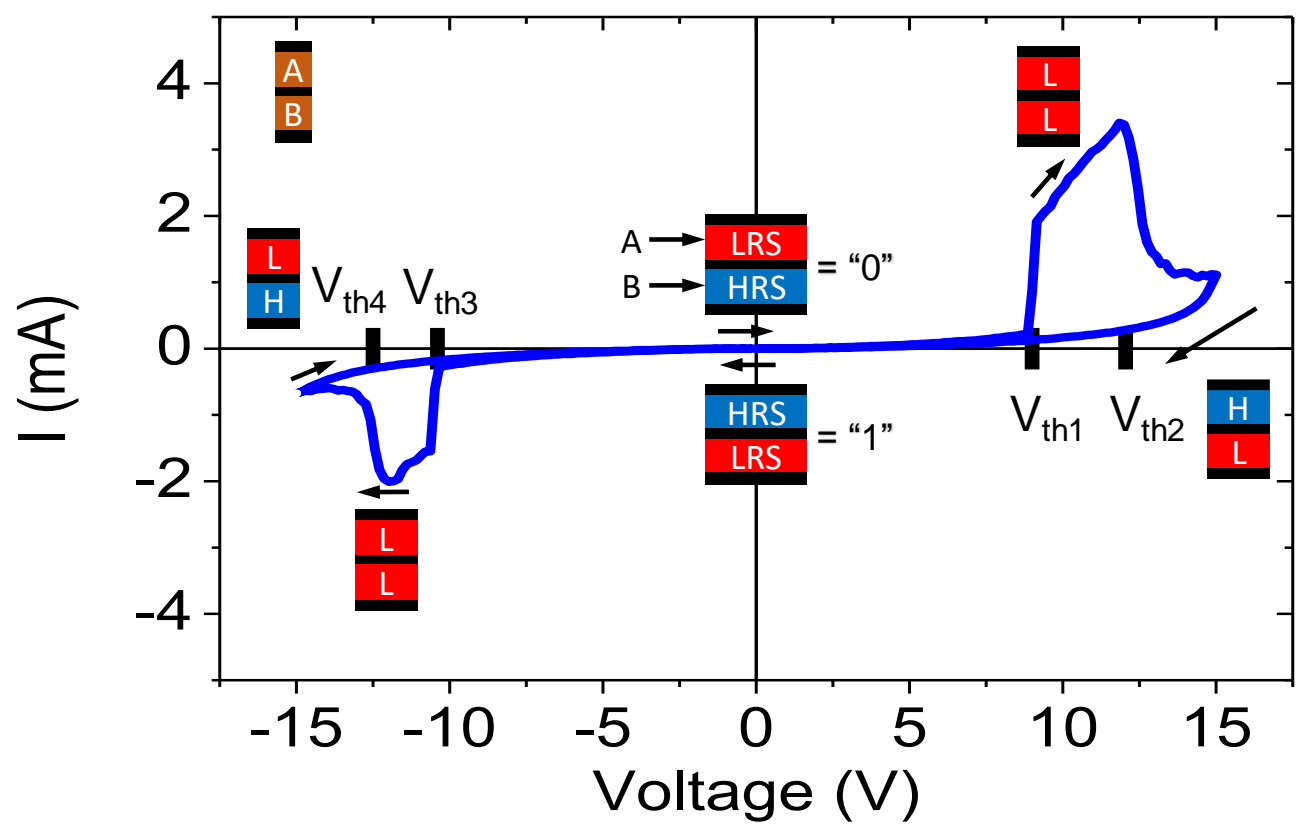

Figure 2. (a) $I-V$ curve of junction A recorded in top-bottom (T-B) contact configuration. (b) $I-V$ of junction $\mathrm{B}$ recorded in reversed $\mathrm{T}-\mathrm{B}$ configuration. (c) $I-V$ curve of device $\mathrm{AB}$ recorded in top-top (T-T) contact configuration. 


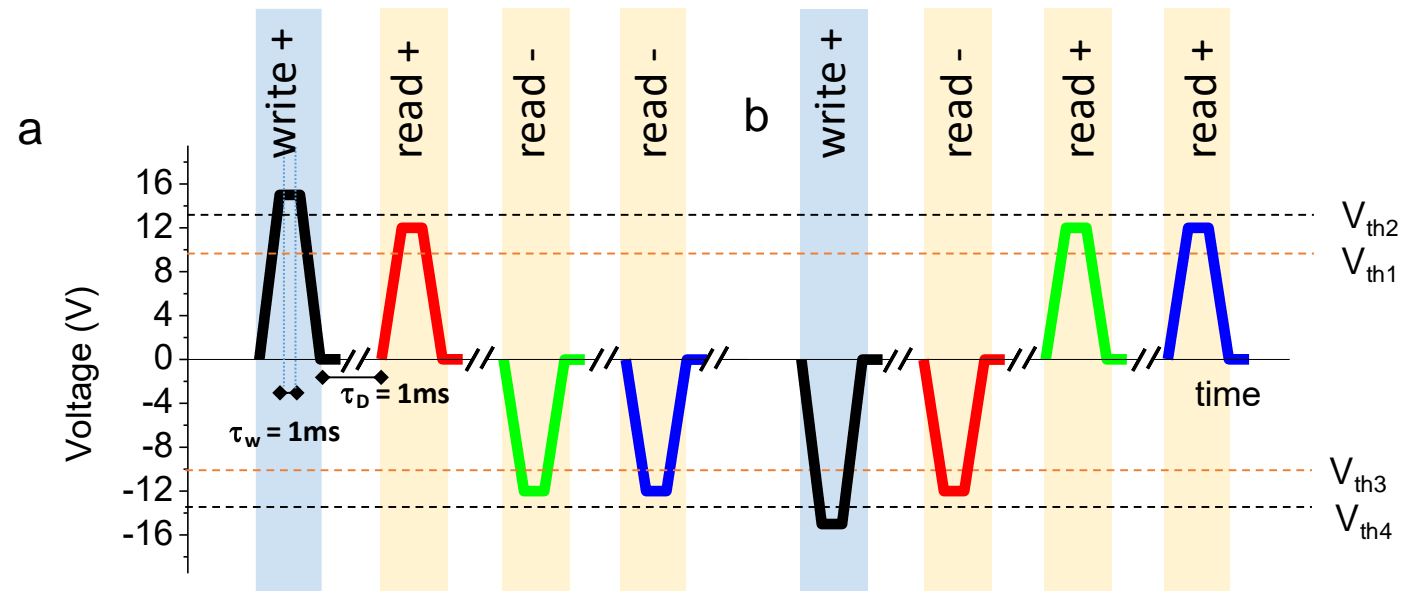

C

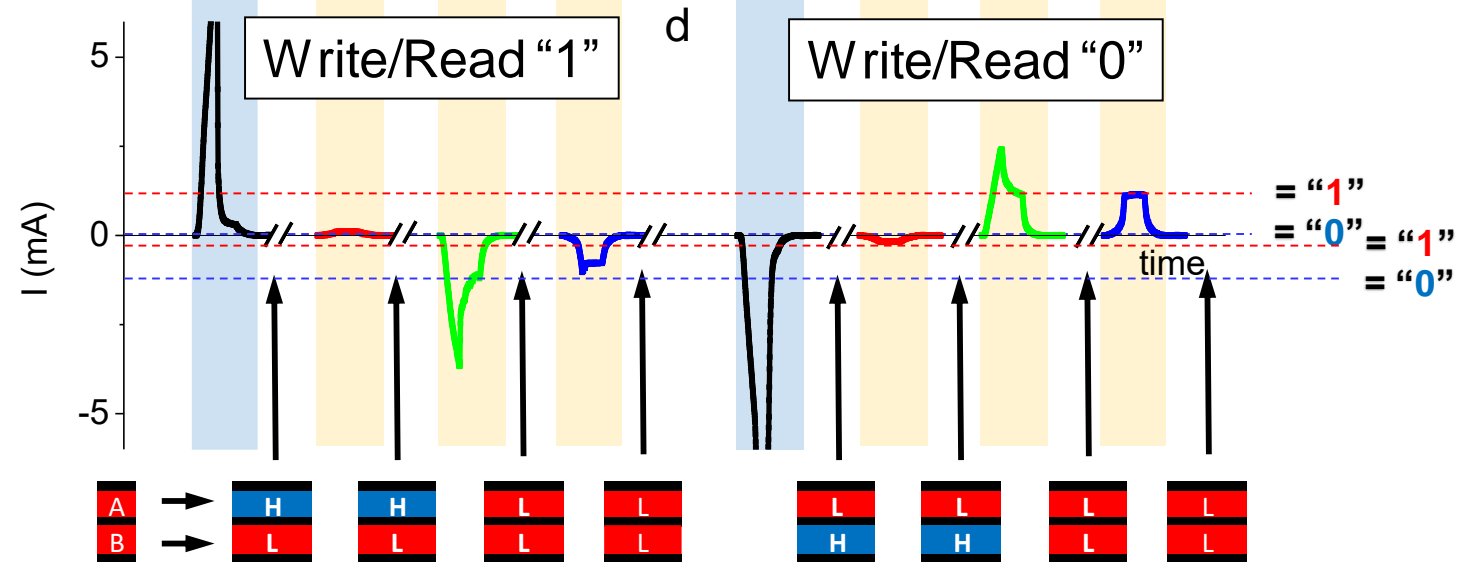

Figure 3. (a) Voltage pulse train used to write and read $\mathrm{HRS}_{\mathrm{A}}-\mathrm{LRS}_{\mathrm{B}}$ (which corresponds to "0"). (b) Voltage pulse train used to write and read LRS $_{A}-H_{R} S_{B}$ (which corresponds to "1"). $(c, d)$ Measured current during application of voltage pulse train shown in $(a, b)$. Background colors indicate if a writing (blue) or reading (yellow) pulse is applied. Top labels also indicate if the write and reading pulses are positive (+) or negative (-). Bottom sketches indicate the resistance states $(\mathrm{H} \equiv \mathrm{HRS}, \mathrm{L} \equiv \mathrm{LRS})$ of the two anti-series connected capacitors. 
a
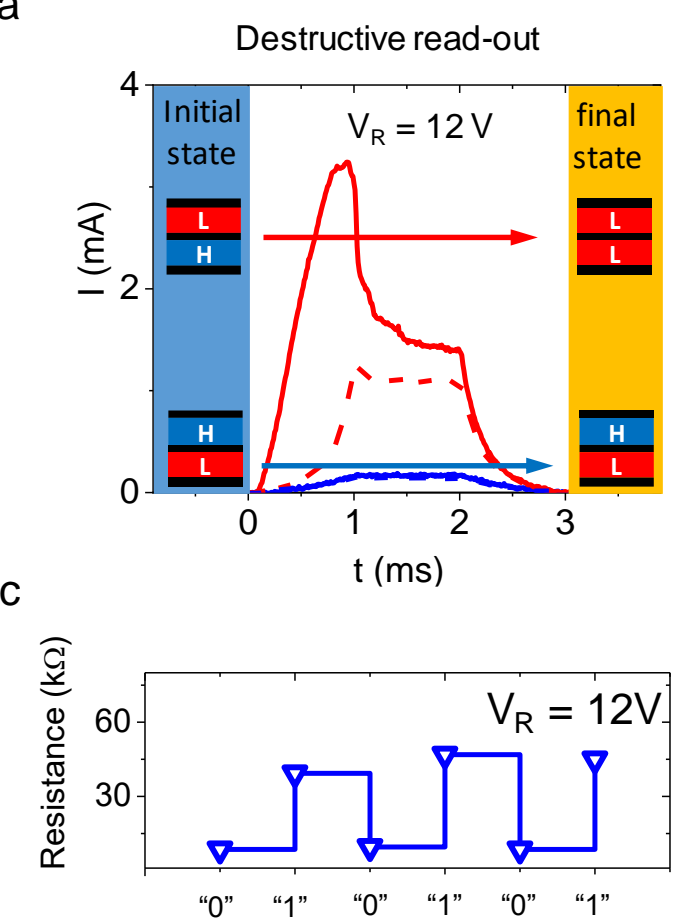

b

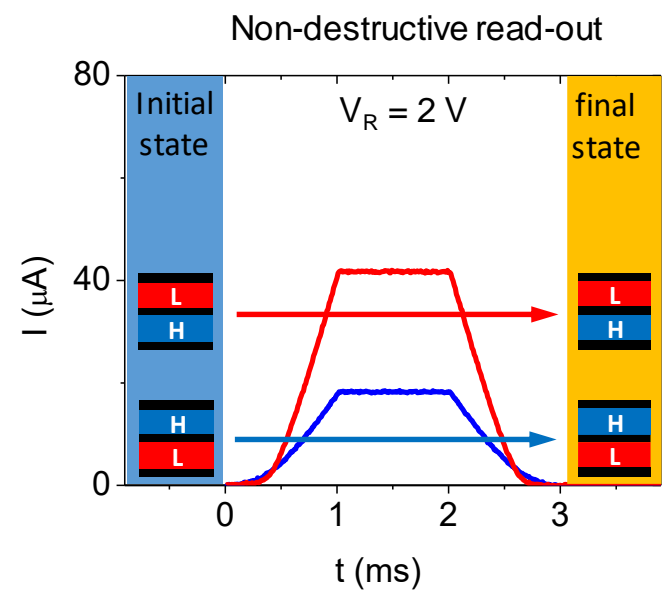

d

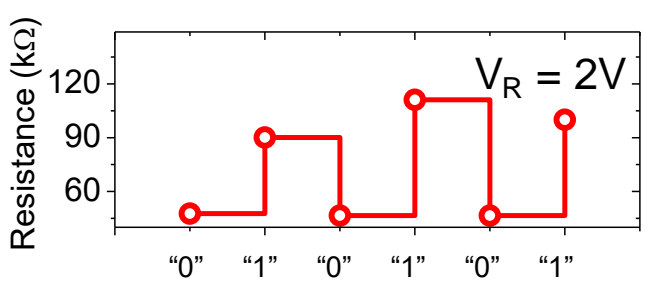

Figure 4. (a) Current recorded during the first and second reading pulses of $V_{R}=+12 \mathrm{~V}$ measured after the writing pulse of $+16 \mathrm{~V}$ (blue) or $-16 \mathrm{~V}$ (red) using $\tau_{D}=1 \mathrm{~s}$ and $\tau_{W, R}=1 \mathrm{~ms}$. (b) Current during the first and second reading pulses of $V_{R}=+1,2$ and $5 \mathrm{~V}$ measured after the writing pulse of $+16 \mathrm{~V}$ (blue) or $-16 \mathrm{~V}$ (red) using $\tau_{D}=1 \mathrm{~s}$ and $\tau_{W, R}=1 \mathrm{~ms}$. Current measured during the first and second pulses overlap. Sketches indicate the resistance states $(\mathrm{H}$ $\equiv \mathrm{HRS}, \mathrm{L} \equiv \mathrm{LRS}$ ) of the two anti-series connected capacitors. (c,d) Resistance states after sequentially setting the " 0 " and " 1 " states, corresponding to $\mathrm{HRS}_{\mathrm{A}}-\mathrm{LRS}_{\mathrm{B}}$ and $\mathrm{LRS}_{\mathrm{A}}-\mathrm{HRS}_{\mathrm{B}}$, respectively, three times using $V_{R}=$ (c) $12 \mathrm{~V}$, (d) $2 \mathrm{~V}$. 
a

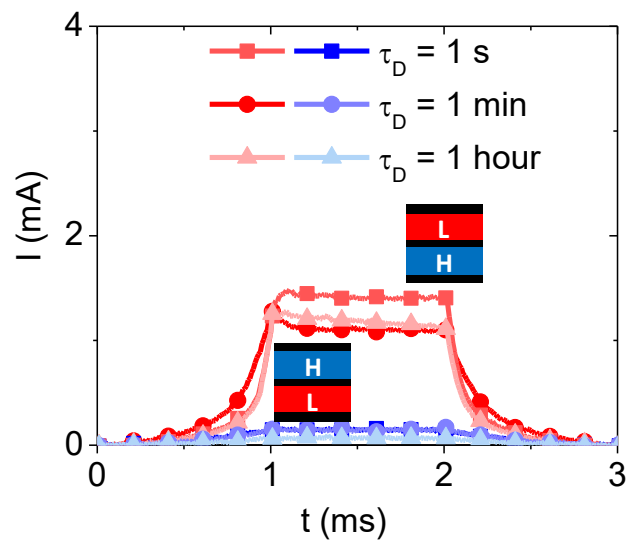

b

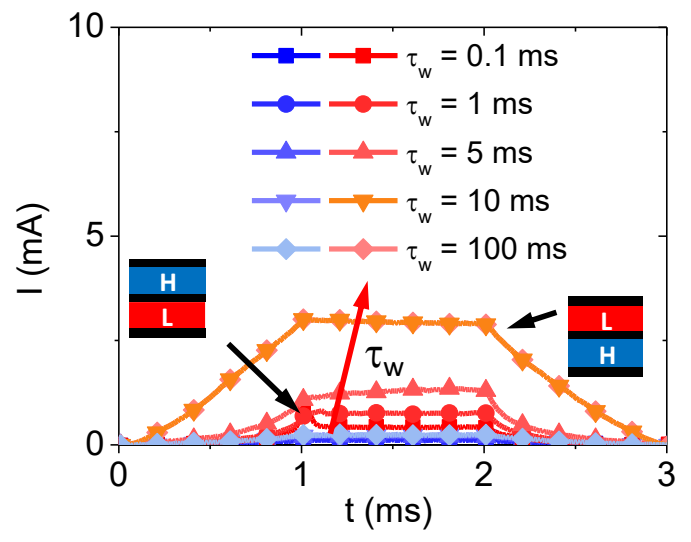

Figure 5. (a) Current during the second reading pulse measured using different delay times $\left(\tau_{D}=1 \mathrm{~s}, 1 \mathrm{~min}\right.$ and $1 \mathrm{~h}$ ) after the writing pulse of $+16 \mathrm{~V}$ (blue) or $-16 \mathrm{~V}$ (red) of $\tau_{W}=1 \mathrm{~ms}$ of duration. (b) Current during the second reading pulse measured after the writing pulse of +16 $\mathrm{V}$ (blue) or $-16 \mathrm{~V}$ (red) of $\tau_{W}=0.1,1,5,10$ and $100 \mathrm{~ms}$ of duration fixing the delay times to $\tau_{D}=1 \mathrm{~s}$. Sketches indicate the resistance states $(\mathrm{H} \equiv \mathrm{HRS}, \mathrm{L} \equiv \mathrm{LRS})$ of the two anti-series connected capacitors. 


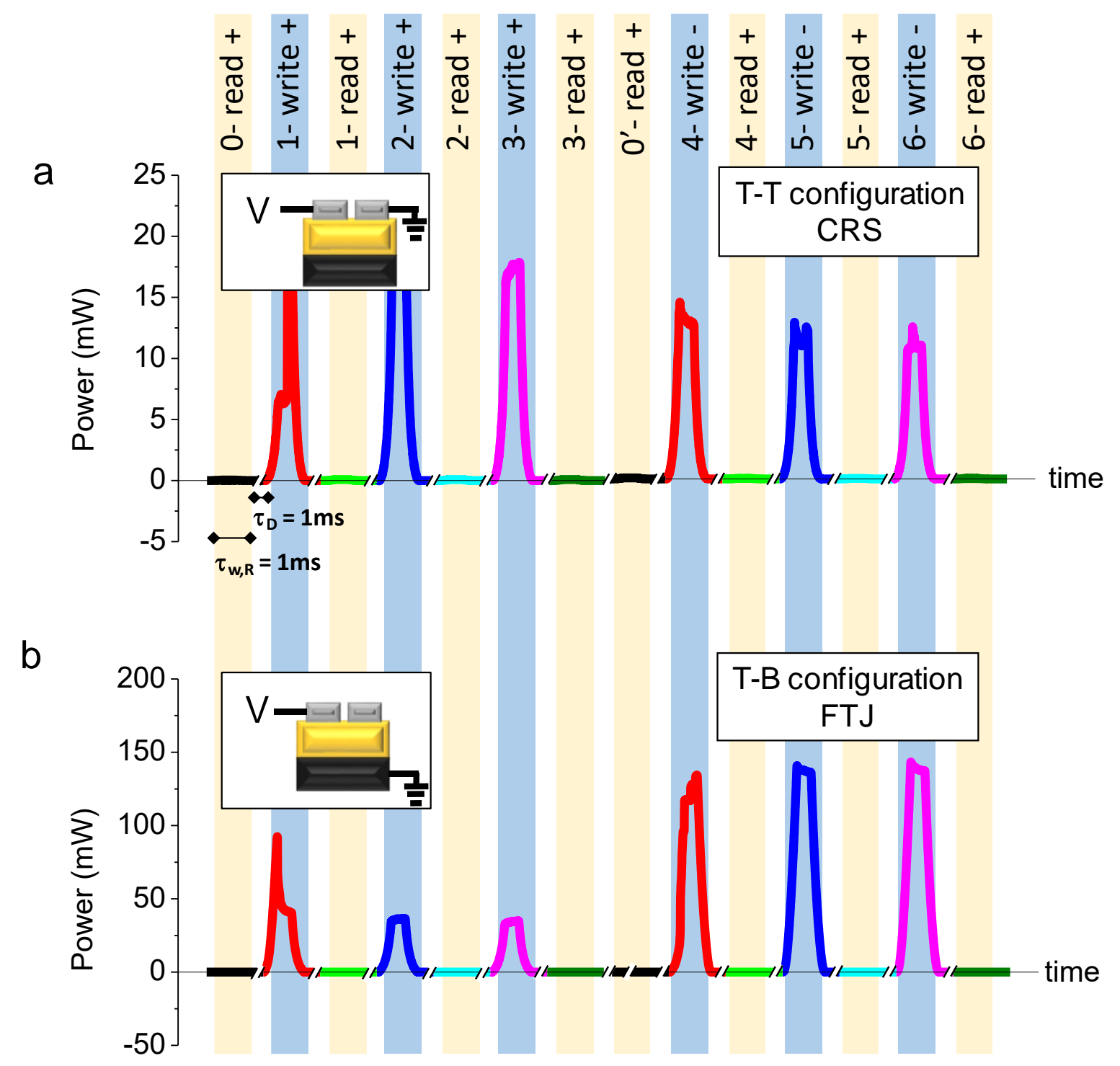

Figure 6. (a) Writing/reading power consumption using the T-T configuration (sketched in the inset). Writing voltage is $\pm 16 \mathrm{~V}$ and reading voltage is $+12 \mathrm{~V}$. (b) Writing/reading power consumption using the T-B configuration (sketched in the inset). Writing voltage is $\pm 15 \mathrm{~V}$ and reading voltage is $+0.1 \mathrm{~V}$. Background colors indicate if a writing (blue) or reading (yellow) pulse is applied. Top labels also indicated if the write/reading pulses are positive $(+)$ or negative (-). 
Table 1. Voltage dependence of the resistance state of the junctions $A$ and $B$, and their connection in anti-serial (indicated as AB). HRS, LRS refer to the resistance states of each single element $(\mathrm{A}, \mathrm{B})$. $\mathrm{HRS}_{\mathrm{CRS}}$ and $\mathrm{LRS}_{\mathrm{CRS}}$ refer to the resistance state of the A-B combination and (" 0 " or " 1 ") to their logic state.

\begin{tabular}{|c|c|c|c|c|c|c|c|c|c|}
\hline V & 0 & $\mathrm{~V}<\mathrm{V}_{\text {th1 }}$ & $V_{\text {th } 1}>V>V_{\text {th } 2}$ & $\mathrm{~V}>\mathrm{V}_{\mathrm{th2}}$ & 0 & $\mathrm{~V}>\mathrm{V}_{\mathrm{th} 3}$ & $\mathrm{~V}_{\text {th } 3}<\mathrm{V}<\mathrm{V}_{\text {th4 }}$ & $V>V_{\text {th4 }}$ & 0 \\
\hline A & LRS & LRS & LRS & HRS & HRS & HRS & LRS & LRS & LRS \\
\hline B & HRS & HRS & LRS & LRS & LRS & LRS & LRS & HRS & HRS \\
\hline$A B$ & $\mathrm{HRS}_{\mathrm{CRS}}$ & $\mathrm{HRS}_{\mathrm{CRS}}$ & LRS $_{\text {CRS }}$ & $\mathrm{HRS}_{\mathrm{CRS}}$ & $\mathrm{HRS}_{\mathrm{CRS}}$ & $\mathrm{HRS}_{\mathrm{CRS}}$ & LRS $_{\text {CRS }}$ & $\mathrm{HRS}_{\mathrm{CRS}}$ & $\mathrm{HRS}_{\mathrm{CRS}}$ \\
\hline State & 0 & & & & 1 & & & & 0 \\
\hline
\end{tabular}




\section{Supporting Information}

\section{Complementary resistive switching using metal-ferroelectric-metal tunnel junctions}

Mengdi Qian, Ignasi Fina, * Florencio Sánchez, and Josep Fontcuberta*

a

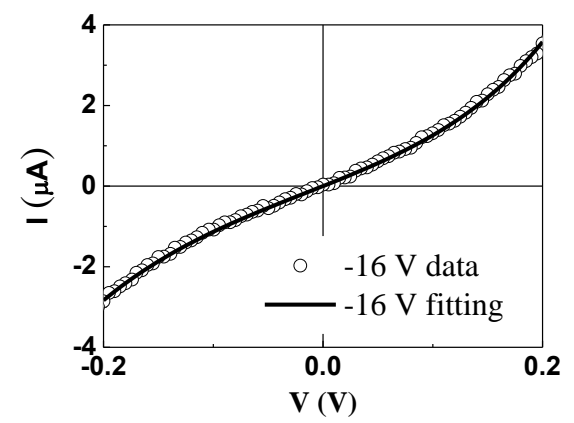

b

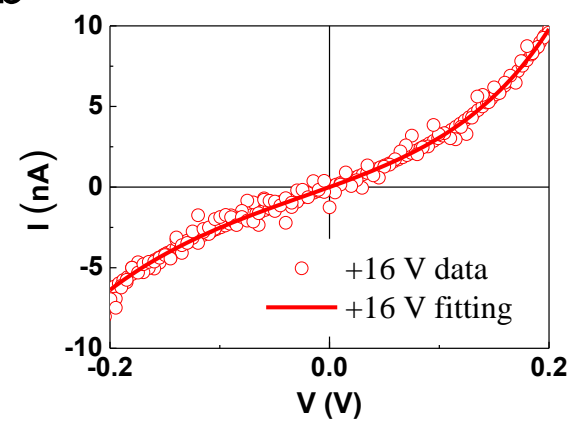

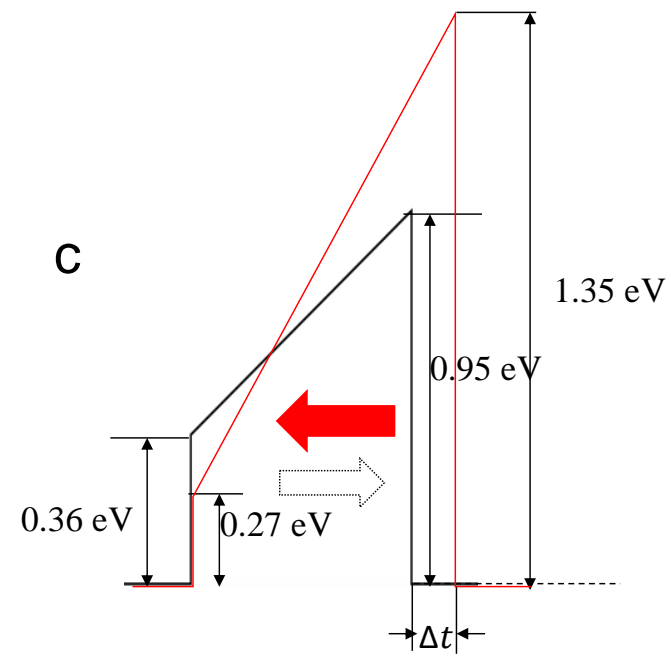

\section{LSMO BTO $\quad$ Pt}

Figure S1. $I-V$ characteristics measured at room-temperature at small voltage range for: (a) LRS and (b) OFF states, respectively. Solid lines across the experimental data are the results of the fits according to the Brinkman model. (c) Sketch of the resulting shape of the barrier in the BTO tunnel junction with the fitting results indicated in the sketch. Black line corresponds to $+16 \mathrm{~V}$ prepoling, and red line to $-16 \mathrm{~V}$. The results indicate a substantial rising of the average barrier height and its asymmetry and barrier thickness in the OFF state. Note that the modification of barrier heights $\varphi_{1}$ and $\varphi_{2}$ corresponding to LSMO/BTO and BTO/Pt interfaces, respectively, which averge increase after $+8 \mathrm{~V}$, which corresponds to polarization pointing towards LSMO. Similar for the effective barrier thickness $(d)$. See the sketch to better visualize it.

The fitting parameters are:

ON state: $\varphi_{1}(\mathrm{ON})=0.95 \mathrm{eV}, \varphi_{2}(\mathrm{ON})=0.36 \mathrm{eV}$ and $d(\mathrm{ON})=2.35 \mathrm{~nm}$,

OFF state: $\varphi_{1}(\mathrm{OFF})=1.35 \mathrm{eV}, \varphi_{2}(\mathrm{OFF})=0.27 \mathrm{eV}$ and $d(\mathrm{OFF})=2.8 \mathrm{~nm}$

The fittings are obtained by minimizing the $\chi^{2}\left[\chi^{2}=\Sigma\right.$ (experimental-fitted) $\left.{ }^{2}\right]$ value accounting for the difference between the experimental values and the fitted ones using the equation: 


$$
\begin{aligned}
& \mathrm{J} \cong C \frac{\exp \left\{\alpha(V)\left[\left(\Phi_{2}-\frac{e V}{2}\right)^{3 / 2}-\left(\Phi_{1}+\frac{e V}{2}\right)^{3 / 2}\right]\right\}}{\alpha^{2}(V)\left[\left(\Phi_{2}-\frac{e V}{2}\right)^{1 / 2}-\left(\Phi_{1}+\frac{e V}{2}\right)^{1 / 2}\right]^{2}} \times \\
& \sinh \left\{\frac{3}{2} \alpha(V)\left[\left(\Phi_{2}-\frac{e V}{2}\right)^{1 / 2}-\left(\Phi_{1}+\frac{e V}{2}\right)^{1 / 2}\right] \frac{e V}{2}\right\}
\end{aligned}
$$

where

$$
\begin{aligned}
& C=-\left(4 e m^{*} m_{e}\right) /\left(9 \pi^{2} \hbar^{3}\right) \\
& \alpha(V) \equiv\left[4 d\left(2 m^{*} m_{e}\right)^{1 / 2}\right] /\left[3 \hbar\left(\Phi_{1}+e V-\Phi_{2}\right)\right]
\end{aligned}
$$

and $m_{e}$ corresponds to the electron mass, $m^{*}$ to its effective mass (fixed to 1 ) and $e$ to its charge.

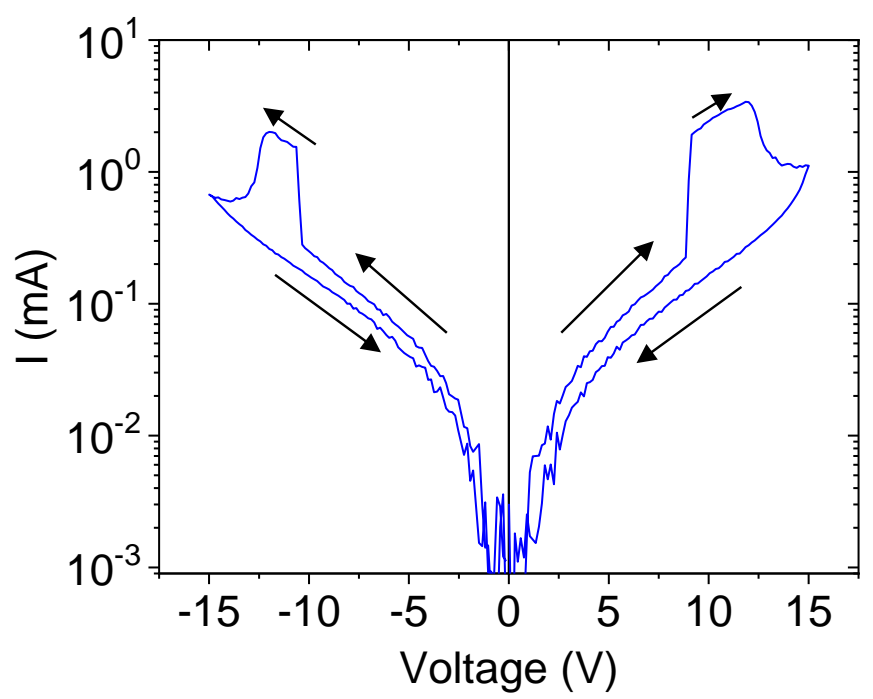

Figure S2. $I-V$ curve in logarithmic scale of the devices measured in $\mathrm{T}-\mathrm{T}$ contact configuration of the AB device shown in Figure 1(c).

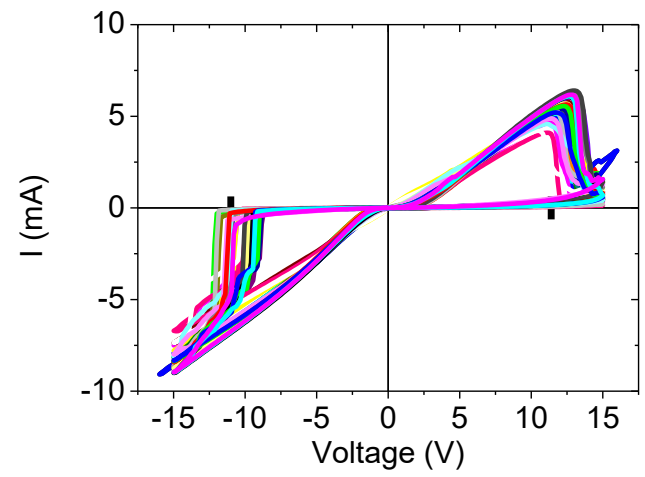

b

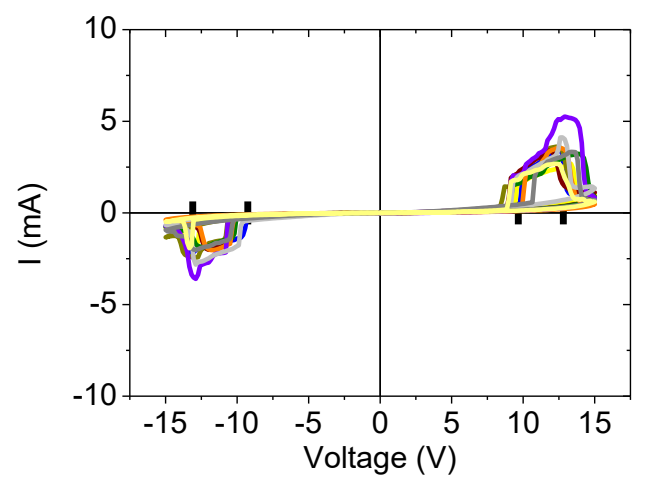

Figure S3. (a) $I-V$ curve of several devices measured in T-B contact configuration across the sample. (b) $I-V$ curve of several devices measured in T-T contact configuration across the sample. 
a

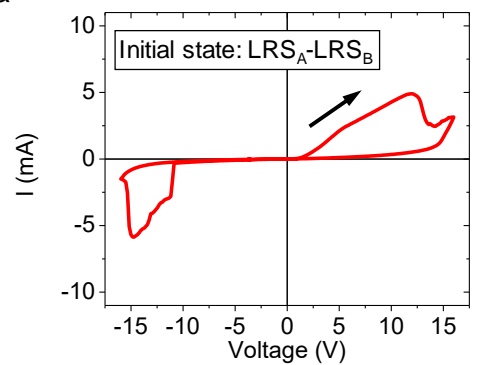

b

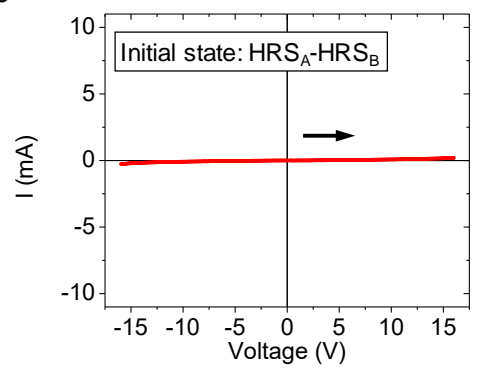

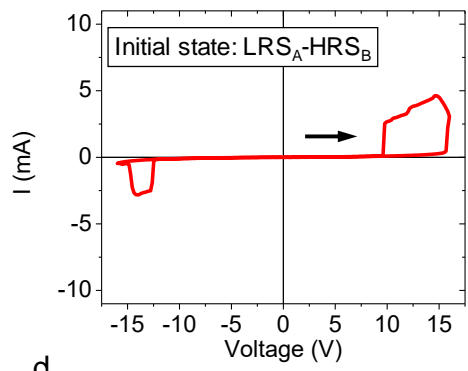

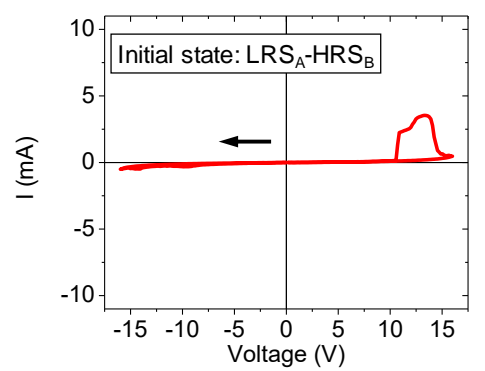

e

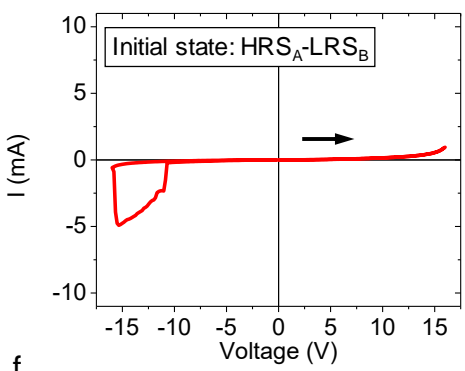

f

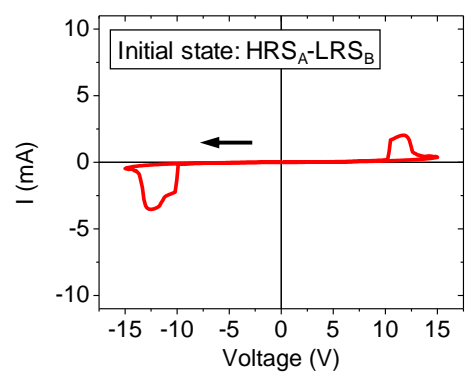

Figure S4. $I-V$ curve measured in T-T contact configuration after preparing the indicated different initial states. In these experiments the different states were prepared separately in junction $\mathrm{A}$ and $\mathrm{B}$ (according to the figure 1 notation) using writing pulses of $\pm 15 \mathrm{~V}$. The voltage excursion used starts at 0 and after voltage is increased or decreased as indicated by the arrow. It can be observed that depending on the different initial state the recorded $I-V$ is different.

a
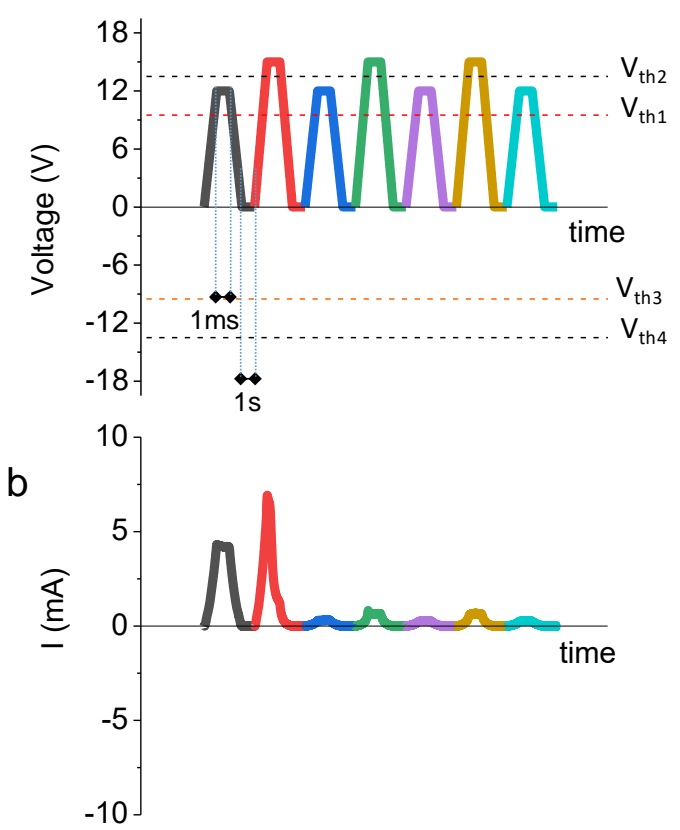

C
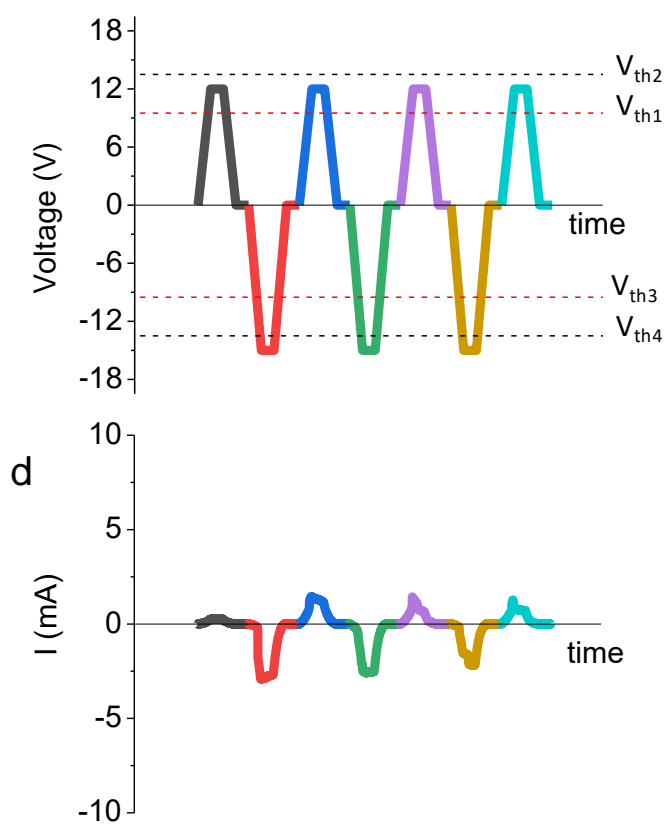

Figure S5. (a) Repetitive write/read voltage pulse train using writing voltages of $+16 \mathrm{~V}$ and reading voltages of $+12 \mathrm{~V}$. (b) Repetitive write/read voltage pulse train using writing voltages of $-16 \mathrm{~V}$ and reading voltages of $+12 \mathrm{~V}$. (c,d) Measured current during the application of voltage pulse train shown in (a) and (b), respectively. It can be observed that repetitive writing produces similar measured current during the reading. 


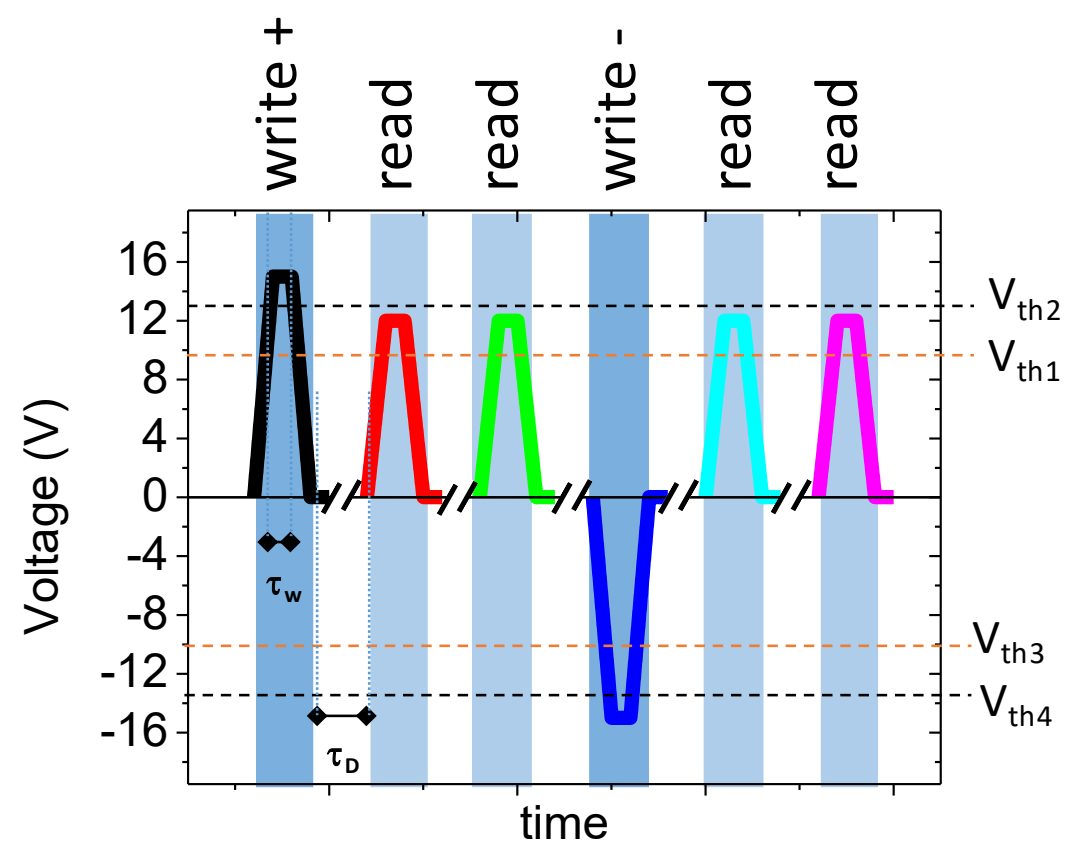

Figure S6. Used write/read voltage pulse train for the experiment shown in Figure 5.

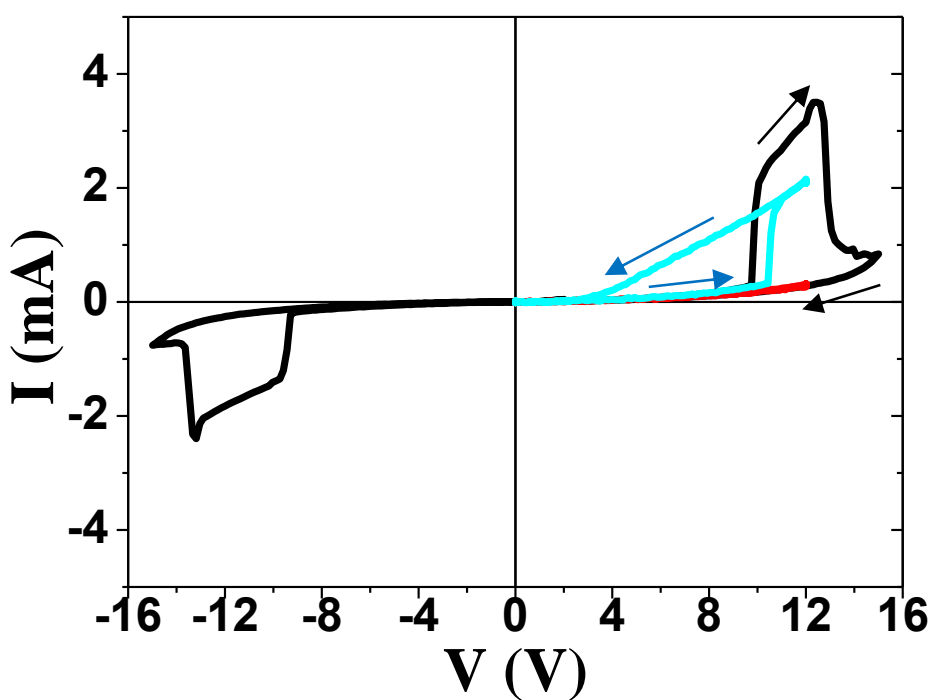

Figure S7. Complete $I-V$ excursion (black), unipolar $I-V$ excursion up to $+12 \mathrm{~V}$ after $V_{W}=+$ $16 \mathrm{~V}$, which settles the $\mathrm{HRS}_{\mathrm{A}}-\mathrm{LRS}_{\mathrm{B}}$ state (red), unipolar $I-V$ excursion up to $+12 \mathrm{~V}$ after $V_{W}=$ - $16 \mathrm{~V}$, which settles the $\mathrm{LRS}_{\mathrm{A}}-\mathrm{HRS}_{\mathrm{B}}$ state (blue). It can be observed that unipolar I-V excursion after $V_{W}=-16 \mathrm{~V}$ produces a high conductive state at remanence. Instead, the unipolar I-V excursion after $V_{W}=+16 \mathrm{~V}$, does not modify the logic state of the device. 
a

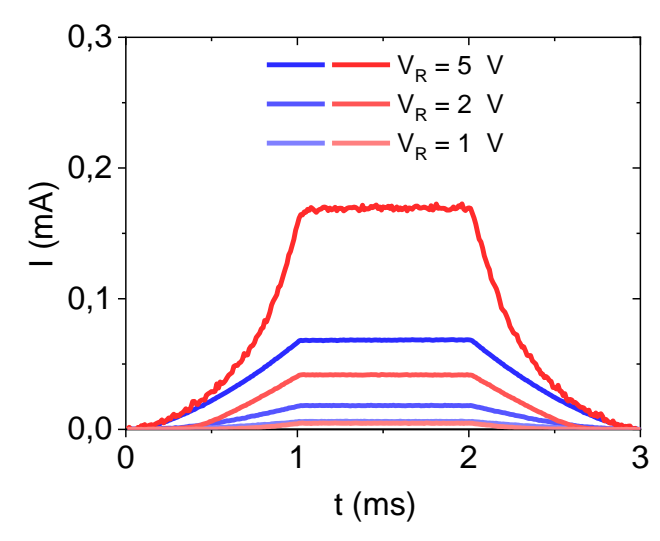

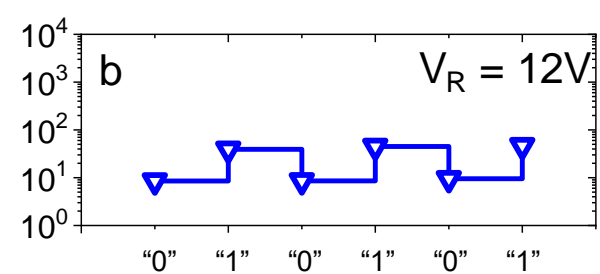
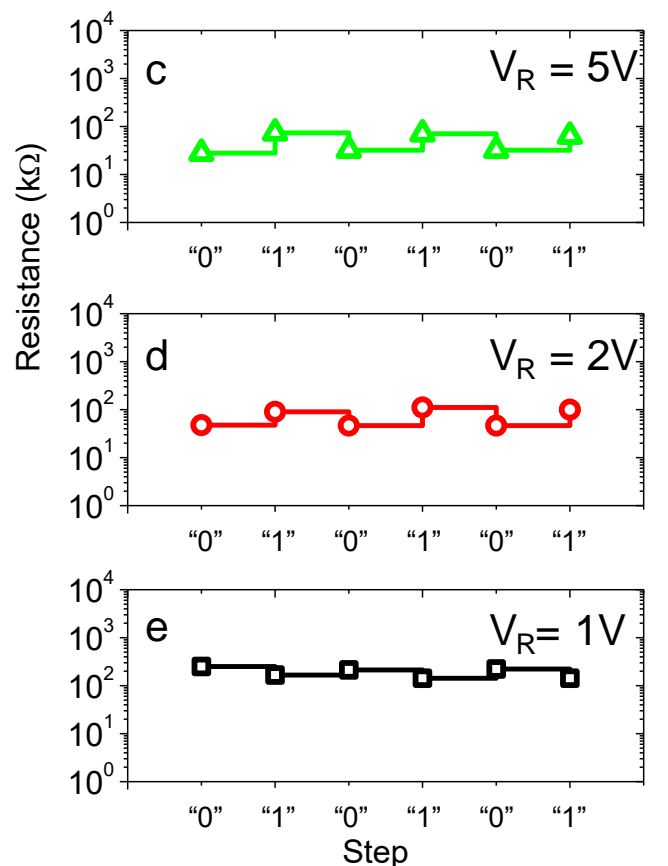

Figure S8. (a) Current during the first and second reading pulses of $V_{R}=+1,2$ and $5 \mathrm{~V}$ measured after the writing pulse of $+16 \mathrm{~V}$ (blue) or $-16 \mathrm{~V}$ (red) using $\tau_{D}=1 \mathrm{~s}$ and $\tau_{W, R}=1 \mathrm{~ms}$. Current measured during the first and second pulses overlap.(b,c,d,e) Resistance states after sequentially setting the " 0 " and "1" states, corresponding to $\mathrm{HRS}_{\mathrm{A}}-\mathrm{LRS}_{\mathrm{B}}$ and $\mathrm{LRS}_{\mathrm{A}}-\mathrm{HRS}_{\mathrm{B}}$, respectively, three times using $V_{R}=$ (b) 12 , (c) 5, (d) 2 and (e) $1 \mathrm{~V}$. It can be observed that the different resistance states are distinguishable down to $V_{R}=2 \mathrm{~V}$

a

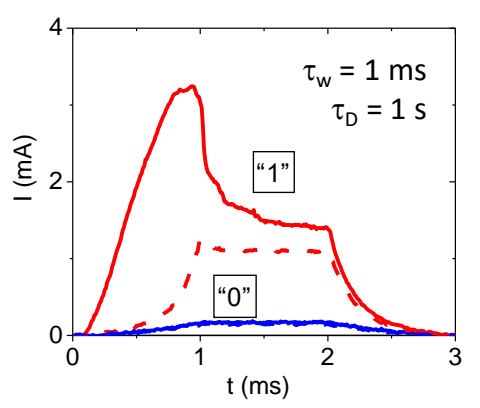

b

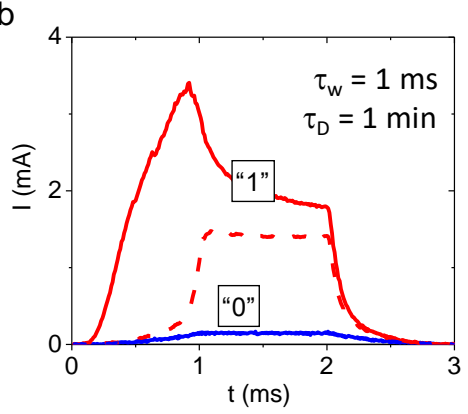

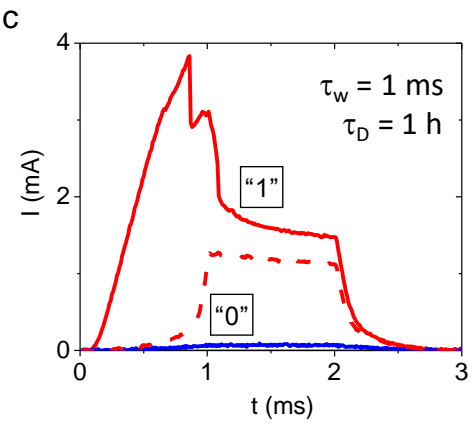

Figure S9. Measured current during the first (solid lines) and the second (dashed lines) reading pulses measured using different delay times $\left(\tau_{\mathrm{D}}=(\mathrm{a}) 1 \mathrm{~s},(\mathrm{~b}) 1 \mathrm{~min}\right.$ and $\left.(\mathrm{c}) 1 \mathrm{~h}\right)$ after the writing pulse of $+16 \mathrm{~V}$ (blue) or $-16 \mathrm{~V}$ (red) of $\tau_{\mathrm{W}}=1 \mathrm{~ms}$ of duration. The voltage pulse train used is that shown in Supp. Inform. Figure 6. It can be observed that during first reading pulse a current spike (not apparent in the second appears). 
a

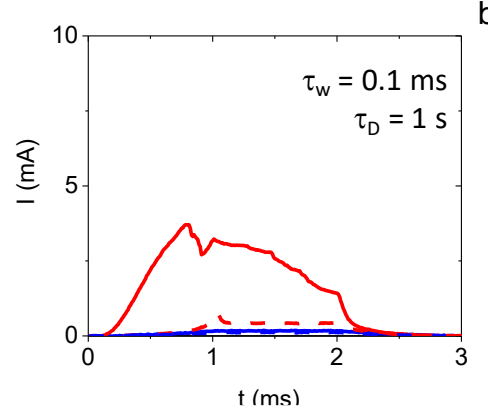

d

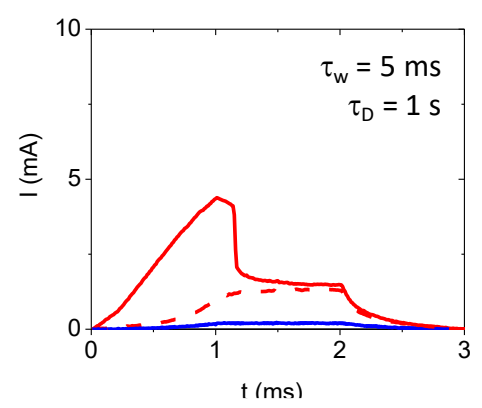

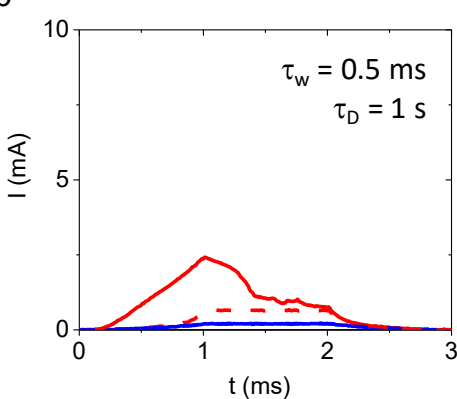

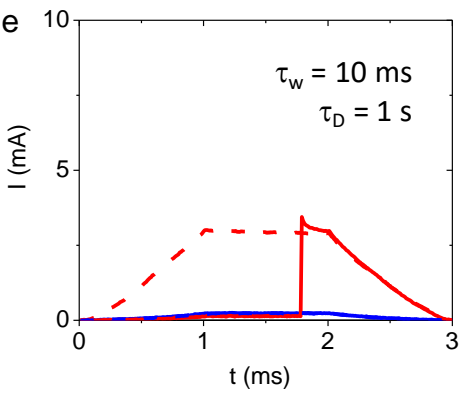

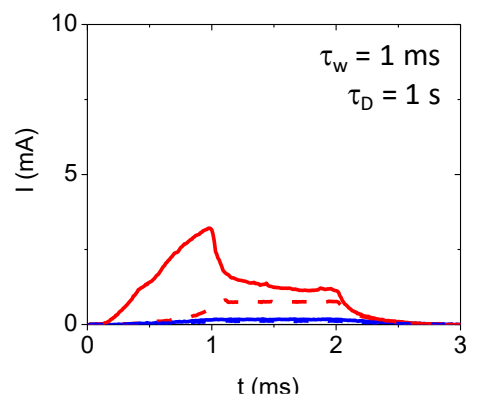

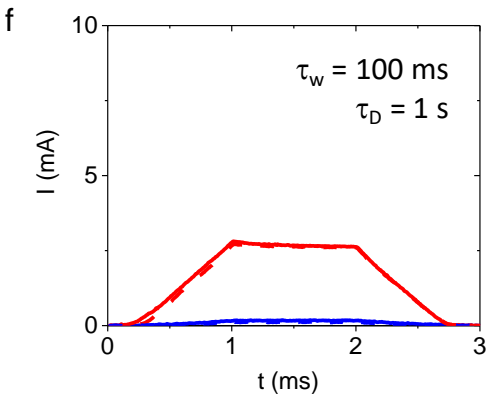

Figure S10. Current measured during the first (solid lines) and second (dashed lines) reading pulses measured after the writing pulse of $+16 \mathrm{~V}$ (blue) or $-16 \mathrm{~V}$ (red) of $\tau_{W}=(\mathrm{a}) 0.1$, (b)0.5, (c)1, (d)10 and (e)100 ms of duration fixing the t delay times to $\tau_{D}=1 \mathrm{~s}$. It can be observed that measured current during the second pulse for $\tau_{W}$ of 10 and $100 \mathrm{~ms}$ is very similar indicating that the contrast between the two states is saturated. It can be observed that during first reading pulse a current spike (not apparent in the second appears).

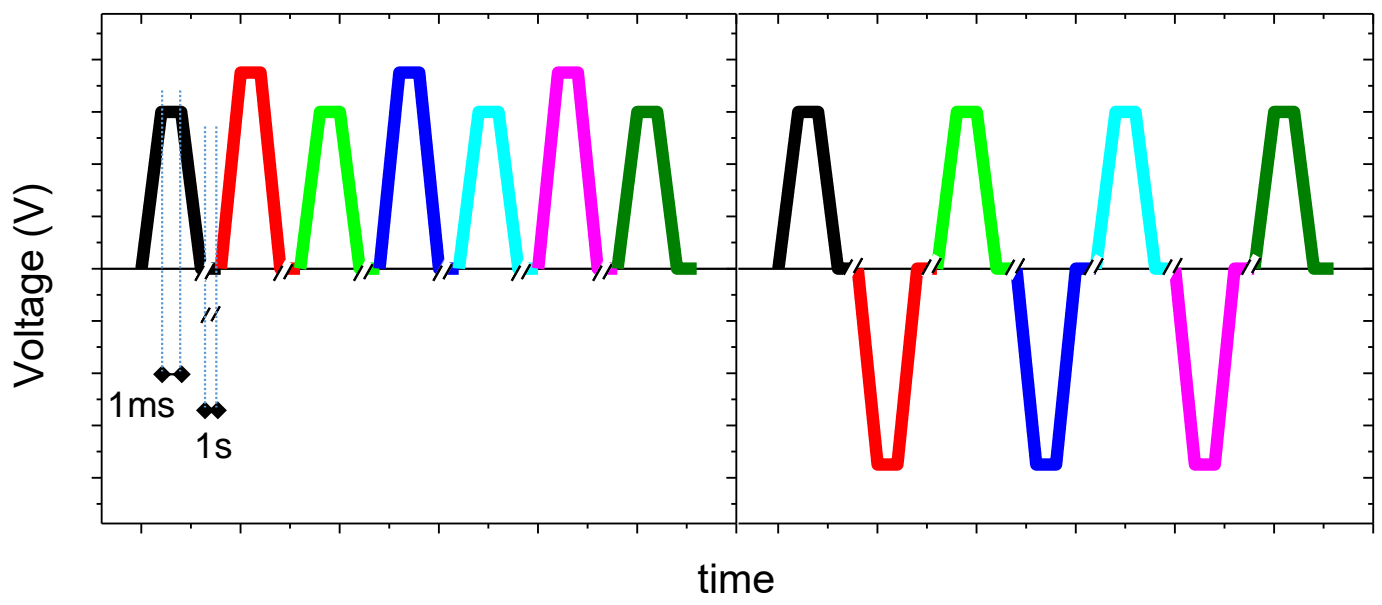

Figure S11. Used write/read voltage pulse train for the experiment shown in Figure 6. 


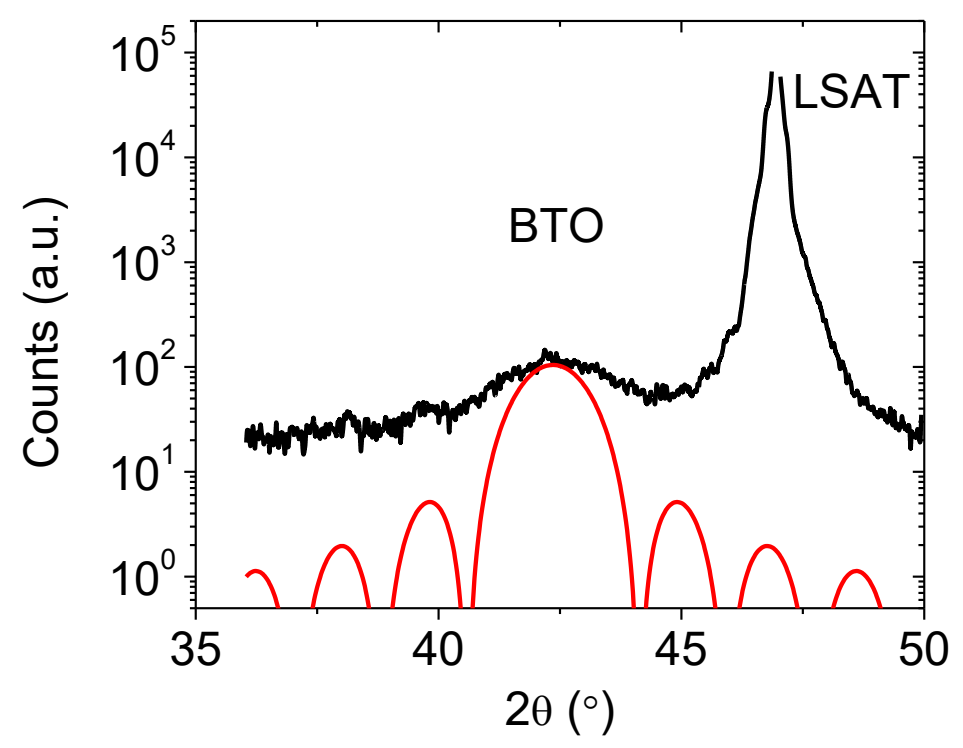

Figure S12. $\theta-2 \theta$ scans around $\mathrm{BTO}(002)$ reflection. Small bumps around the BTO peak are ascribed to Laue oscillations. The Lau simulation shown below (read line) correspond to a infinitely wide BTO layer using out of plane parameter $4.268 \AA$ and a thickness of 12.5 unit cells (corresponding to $5.3 \mathrm{~nm}$ ) which is closely coincident with the nominal film thickness.

a

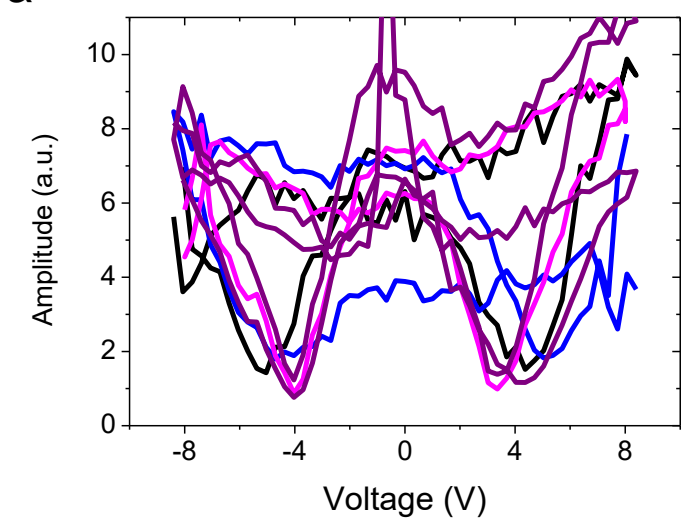

b

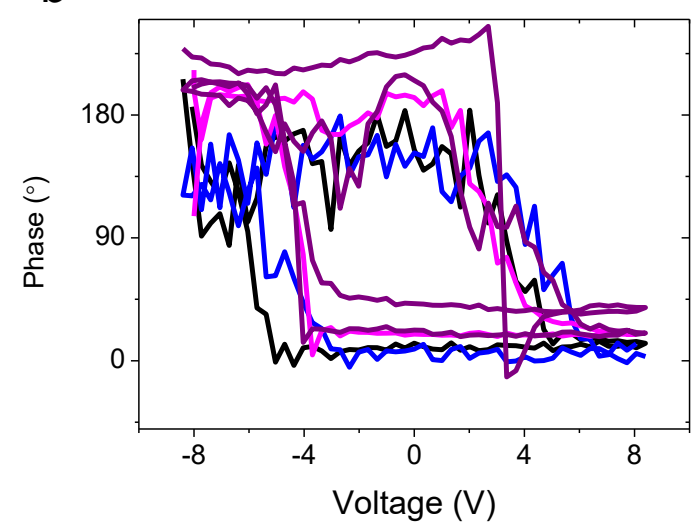

Figure S13. PFM force microscopy measurements performed at different positions of a BTO $(4 \mathrm{~nm})$ film grown on a STO substrate using the same nominal conditions of the sample characterized in the manuscript. (a, b) Amplitude and phase respectively of the piezoresponse versus applied voltage. Experimental details: Measurements are performed with a MFP-3D Asylum Research microscope. MikroMasch silicon cantilevers with Pt coating (ANSCM-PT) are used. To achieve better sensitivity due to the low amplitude of the piezoresponse signal, the dual AC resonance tracking (DART) method is employed. ${ }^{[1,2]}$ PFM voltage hysteresis loops are always performed at remanence using a dwell time of $100 \mathrm{~ms}$. The butterfly shape of the amplitude versus voltage loop and the $180^{\circ}$ phase contrast in the phase versus voltage loop shows the ferroelectric nature of the sample. Similar experiments in LSAT sample, with a coercive voltage above $10 \mathrm{~V}$ as revealed by Figure 2(a,b), are not possible due to PFM set-up maximum applied voltage is $10 \mathrm{~V}$. 